\title{
Osculating properties of decomposable scrolls
}

\author{
Antonio Lanteri*1 and Raquel Mallavibarrena**2 \\ ${ }^{1}$ Dipartimento di Matematica "F. Enriques", Università degli Studi di Milano, Via C. Saldini, 50, I-20133 Milano, Italia \\ ${ }^{2}$ Departamento de Algebra, Facultad de Matemáticas, Universidad Complutense de Madrid, Ciudad Universitaria, E-28040 \\ Madrid, Spain
}

Key words Scroll (non-normal); osculating space; inflectional locus; (higher) discriminant locus. MSC (2000) Primary: 14F05, 14N05; Secondary: 14J26, 14J40, 14C20, 53A20

Osculating spaces of decomposable scrolls (of any genus and not necessarily normal) are studied and their inflectional loci are related to those of their generating curves by using systematically an idea introduced by Piene and Sacchiero in the setting of rational normal scrolls. In this broader setting the extra components of the second discriminant locus -deriving from flexes- are investigated and a new class of uninflected surface scrolls is presented and characterized. Further properties related to osculation are discussed for (not necessarily decomposable) scrolls.

\section{Introduction}

The inflectional behavior of a projective variety belongs to its extrinsic geometry. In particular, flexes can appear on projective manifolds under (isomorphic) projections. Though this observation is obvious, it seems that several projective manifolds have been extensively investigated from the point of view of their osculatory behavior only in the linearly normal case. This is true e.g., for rational scrolls of any dimension [9] and also for elliptic surface scrolls [7]. In this paper we mainly consider decomposable scrolls, not necessarily linearly normally embedded, and we study their inflectional behavior.

Decomposable scrolls $X \subset \mathbb{P}^{N}$, whose construction generalizes that of rational normal scrolls, are generated by $n$ curves $C_{i}(i=1, \ldots, n)$ isomorphic each other, lying in linearly independent linear subspaces generating the whole $\mathbb{P}^{N}$ (see Section 1). They are very well suited to investigate their $k$-th inflectional loci $\Phi_{k}(X)$. We do that developing systematically the local description used in [9] and [7], and in Sections 1 and 2 we succeed to describe several properties of $\Phi_{k}(X)$, relating them to the inflectional loci of the generating sections $C_{i}$.

In particular, restricting to the case of rational non-normal scrolls our approach allows us to produce in Section 3 a new series of counterexamples to the even dimensional part of a conjecture of Piene and Tai [10]. While the odd dimensional part of this conjecture has been proved several years ago [10], [3], the even dimensional part is false for certain linearly normal scrolls, as shown by the first author [6]. However we want to stress that the new counterexamples exhibited here are rational scrolls, though, of course, not linearly normal. We also characterize these examples in the framework of decomposable scrolls (Theorem 3.4). This adds some information in order to correct the even dimensional part of the conjecture.

Let $X$ be a decomposable scroll. While describing $\Phi_{k}(X)$ for $k>2$ involves inflectional loci of lower order, the description becomes very easy for $k=2$. In particular, we show that for a decomposable scroll $X, \Phi_{2}(X)$ can have only two types of irreducible components. Let $G$ be any such a component. Then, either $G$ is a sub-fibre of a fibre of $X$, or $X$ is rational, some curve $C_{i}$ is a line, and $G$ is a sub-scroll of $X$ given by a Segre product (Proposition 4.2).

This precise description of $\Phi_{2}(X)$ allows us to study in Section 4 the second discriminant locus of a decomposable scroll $X \subset \mathbb{P}^{N}$. This is the Zariski closed subset $\mathcal{D}$ of $\mathbb{P}^{N \vee}$ parameterizing all hyperplane sections of $X$ admitting a triple point. The main component of $\mathcal{D}$ is the second dual variety of $X$, which parameterizes

\footnotetext{
* Corresponding author: e-mail: lanteri@mat.unimi.it, Phone: +3902 50316153, Fax: +390250316090

** e-mail: rmallavi@mat.ucm.es, Phone: +34913944657, Fax: +34913944607
} 
osculating hyperplanes to $X$ at general points and their limits. But when $X$ has flexes, extra components $\mathcal{D}_{G}$ of $\mathcal{D}$ arise, coming from the irreducible components $G$ of $\Phi_{2}(X)$. Our study of $\Phi_{2}(X)$ allows us to describe these components: either $\mathcal{D}_{G}$ is a linear space or it is a 1-dimensional family of linear spaces. In particular, we show that $\mathcal{D}_{G}$ is a scroll if and only if $X$ is a rational normal scroll generated by some lines plus conics and/or twisted cubics (Example 4.3 and Proposition 4.4). Moreover, we characterize rational normal scrolls generated by some lines plus some conics as the decomposable scrolls admitting an irreducible component $\mathcal{D}_{G}$ of $\mathcal{D}$ which is a rational normal scroll (Theorem 4.7).

In Section 5, we come to surface scrolls, not necessarily linearly normal, regardless the fact they are decomposable or not. Here the techniques developed in the previous sections fail. We discuss two points arising from [6]. a) Indecomposable elliptic surface scrolls of invariant -1 have been studied in [6]. By adapting the approach used there, we investigate those of invariant 0 , providing a description of their flexes in terms of base points of suitable linear systems related to the one giving the embedding (Proposition 5.1). b) The lowest dimension of any osculating space to a surface scroll is 3, as shown in [6]. Moreover, Example 3.2 shows that any higher order osculating space can have very low dimension at some points. Here we find sufficient conditions to grant that all $k$-th osculating spaces of a surface scroll have dimension $\geq k+1$. They are formulated in terms of the (relatively) good properties of the linear system giving rise to the embedding (Theorem 5.2).

\section{Notation and background}

We work over the field of complex numbers. Let $X$ be a smooth projective variety of dimension $n \geq 1$. If $L$ is a line bundle on $X$ we denote by $|W|$ the (not necessarily complete) linear system defined by a vector subspace $W \subseteq H^{0}(X, L)$. Suppose that $|W|$ is very ample, i. e., the map defined by $W$ is an embedding $\varphi_{W}: X \hookrightarrow \mathbb{P}(W)=\mathbb{P}^{N}$. Then $L=\varphi_{W}^{*}\left(\mathcal{O}_{\mathbb{P}^{N}}(1)\right)$. In this case, frequently we look at the pair $(X, W)$, or at the triplet $(X, L, W)$, in place of the non-degenerate embedded variety $\varphi_{W}(X) \subset \mathbb{P}^{N}$ and sometimes we do not distinguish between $X$ and its image.

For any integer $k \geq 0$ let $J_{k} L$ be the $k$-th jet bundle of $L$. For every $x \in X$ we denote by

$$
j_{k, x}^{(X, W)}: W \rightarrow\left(J_{k} L\right)_{x}
$$

the homomorphism associating to every section $\sigma \in W$ its $k$-th jet evaluated at $x$. When the subspace $W$ we are dealing with is clear from the context, or the discussion involves a single pair $(X, W)$, we simply write $j_{k, x}^{X}$ or $j_{k, x}$ respectively, instead of $j_{k, x}^{(X, W)}$. Recall that $j_{k, x}(\sigma)$ is represented in local coordinates by the Taylor expansion of $\sigma$ at $x$, truncated after the order $k$. So, if $|W|$ is very ample, the $k$-th osculating subspace to $X$ at a point $x \in X$ is defined as $\operatorname{Osc}_{x}^{k}(X):=\mathbb{P}\left(\operatorname{Im} j_{k, x}^{(X, W)}\right)$. Identifying $\mathbb{P}^{N}$ with $\mathbb{P}(W)$ (the set of codimension 1 vector subspaces of $W$ ) we see that $\operatorname{Osc}_{x}^{k}(X)$ is a linear subspace of $\mathbb{P}^{N}$. To avoid that it fills up the whole ambient space we assume that $N$ is large enough. For instance, to discuss osculation for surfaces, i. e., $k=n=2$, a reasonable assumption is that $N \geq 6$ or even 5 , depending on the regularity of the surface we are dealing with. Recalling that $\operatorname{rk}\left(J_{k} L\right)=\left(\begin{array}{c}k+n \\ n\end{array}\right)-1$ we have $\operatorname{dim}\left(\operatorname{Osc}_{x}^{k}(X)\right) \leq \min \left\{N,\left(\begin{array}{c}k+n \\ n\end{array}\right)-1\right\}$. Let $\mathcal{U} \subseteq X$ be the Zariski dense open subset where the rank of the homomorphism $j_{k, x}^{(X, W)}: W \rightarrow\left(J_{k} L\right)_{x}$ attains its maximum, say $s(k)+1$. The $k$-th inflectional locus of $(X, W)$ is defined by $\Phi_{k}(X)=X \backslash \mathcal{U}$. So $x \in \Phi_{k}(X)$ if and only if $\operatorname{dim}\left(\operatorname{Osc}_{x}^{k}(X)\right)<s(k)$. By flex we simply mean a point in $\Phi_{2}(X)$, while a higher flex is a point of $\Phi_{k}(X)$ with $k>2$. We say that $X$ is uninflected to mean that $\Phi_{2}(X)=\emptyset$. Of course $\Phi_{h}(X) \subseteq \Phi_{k}(X)$ for $h \leq k$. Let $n=1$. If $N<k$, then clearly $\Phi_{k}(X)=X$. However, if $N \geq k$ then $\Phi_{k}(X) \subsetneq X$ (e. g., see [1, p. 37, Ex C-2]). In particular, $\Phi_{N}(X)=\emptyset$ if and only if $X$ is a rational normal curve [1, p. 39, Ex C-14].

Now let $x \in \mathcal{U}$. A hyperplane $H \in \mathbb{P}^{N \vee}$ is said to be $k$-th osculating to $X$ at $x$ if $H \supseteq \operatorname{Osc}_{x}^{k}(X)$. Then the $k$-th dual variety $X_{k}^{\vee}$ of $(X, W)$ is defined as the closure in $\mathbb{P}^{N \vee}$ of the locus parameterizing all $k$-th osculating hyperplanes to $X$ at points of $\mathcal{U}$.

By scroll we mean an embedded smooth projective variety $Y \subset \mathbb{P}^{N}$ of dimension $n \geq 1$ endowed with a morphism $\pi: Y \rightarrow C$ over a smooth curve $C$ such that $\left(f,\left.\mathcal{O}_{\mathbb{P}^{N}}(1)\right|_{f}\right)=\left(\mathbb{P}^{n-1}, \mathcal{O}_{\mathbb{P}^{n-1}}(1)\right)$ for every fibre $f$ of $\pi$, or the corresponding pair $(X, W)$ with $|W|$ very ample, such that $Y=\varphi_{W}(X)$. Of course $X=C$ if $n=1$. We need to fix some more notation. 
Let $(X, W)$ be a scroll. As is known, for any $k \geq 2$ we have a strict inequality $\operatorname{dim}\left(\operatorname{Osc}_{x}^{k}(X)\right)<\left(\begin{array}{c}k+n \\ n\end{array}\right)-1$ at every point $x \in X$. In fact, there are local coordinates $\left(u, v_{2}, \ldots, v_{n}\right)$ around every point $x \in X$ such that the homogeneous coordinates $x_{i}(i=0, \ldots, N)$ of the points of the variety locally can be written as $x_{i}=$ $a_{i}(u)+\sum_{j=2}^{n} v_{j} b_{i j}(u)$, where $a_{i}$ and $b_{i j}$ are holomorphic functions of $u$. Since every section $\sigma \in W$ is a linear combination $\sigma=\sum_{i=0}^{N} \lambda_{i} x_{i}$ we thus see that the second derivatives $\sigma_{v_{j} v_{h}}$ vanish at every point. Then $\operatorname{dim}\left(\operatorname{Osc}_{x}^{2}(X)\right) \leq 2 n$, and differentiating further up to the order $k$ we see that

$$
\operatorname{dim}\left(\operatorname{Osc}_{x}^{k}(X)\right) \leq n k \quad \text { for every } x \in X .
$$

Finally, we set $\mathbb{F}_{e}=\mathbb{P}\left(\mathcal{O}_{\mathbb{P}^{1}} \oplus \mathcal{O}_{\mathbb{P}^{1}}(-e)\right)$ to denote the Segre-Hirzebruch surface of invariant $e(e \geq 0)$. Then, as in [5. p. 372], $C_{0}$ stands for a section of minimal self-intersection and $f$ for a fibre.

\section{Decomposable scrolls and their flexes}

The situation we consider for the most part of this paper is inspired by that in [9] and [7, Sec. 2]. Let $C$ be a smooth curve of genus $g$. For $i=1, \ldots, n$ let $\mathcal{L}_{i}$ be a very ample line bundle on $C$ and let $V_{i} \subseteq H^{0}\left(C, \mathcal{L}_{i}\right)$ be a vector subspace such that $\left|V_{i}\right|$ gives rise to an embedding

$$
\varphi_{i}: C \rightarrow \mathbb{P}^{r_{i}}=\mathbb{P}\left(V_{i}\right) .
$$

Set $C_{i}=\varphi_{i}(C)$. Let $V=\oplus_{i=1}^{n} V_{i}, \mathcal{E}=\oplus_{i=1}^{n} \mathcal{L}_{i}$ and consider the projective bundle $P=\mathbb{P}(\mathcal{E})$. By identifying $V$ with a vector subspace of $H^{0}(P, L)$, where $L$ is the tautological line bundle on $P$, we get an embedding

$$
\varphi: P \rightarrow \mathbb{P}^{N}=\mathbb{P}(V) .
$$

We set $X=\varphi(P)$. According to [7, p. 151] we say that $X$ is the decomposable scroll generated by $C_{1}, \ldots, C_{n}$. For a point $p \in C$, let $p_{i}=\varphi_{i}(p) \in C_{i}$. Geometrically, $X$ is generated by the linear spaces $f_{p}:=\left\langle p_{1}, \ldots, p_{n}\right\rangle \cong$ $\mathbb{P}^{n-1}$ as the point $p$ varies on $C$; note that all the linear spans $\left\langle C_{i}\right\rangle=\mathbb{P}^{r_{i}}$ of the $C_{i}$ 's are skew each other and generate the whole ambient space $\mathbb{P}^{N}$. Let $t$ be a local parameter on $C$ such that $\varphi_{i}(p)=\left(x_{0}(0), \ldots, x_{r_{i}}(0)\right)$ corresponds to $t=0$. Locally, around $p$, the homomorphism $j_{k}^{C_{i}}: V_{i} \rightarrow J_{k} \mathcal{L}_{i}$ is represented by the matrix

$$
M_{k}^{i}(t)=\left(\begin{array}{cccc}
x_{0}(t) & x_{1}(t) & \ldots & x_{r_{i}}(t) \\
x_{0}^{\prime}(t) & x_{1}^{\prime}(t) & \ldots & x_{r_{i}}^{\prime}(t) \\
\cdot & \cdot & \ldots & \cdot \\
\dot{v} & \dot{\cdot} & \ldots & \dot{\cdot} \\
x_{0}^{(k)}(t) & x_{1}^{(k)}(t) & \ldots & x_{r_{i}}^{(k)}(t)
\end{array}\right) .
$$

The linear space spanned by the row vectors of the matrix $M_{k}^{i}(0)$ defines the $k$-th osculating space to $C_{i}$ at $p_{i}$. Note that, if $k>r_{i}$, then every $k$-th osculating space to $C_{i}$ is the whole space $\mathbb{P}^{r_{i}}=\mathbb{P}\left(V_{i}\right)$. Now let $\lambda_{1}, \ldots, \lambda_{n}$ denote homogeneous coordinates corresponding to a local trivialization of $\mathcal{E}$ around $p$, and, for $\lambda_{n} \neq 0$, set $v_{i}=\lambda_{i} / \lambda_{n}$. Then $\left(t, v_{1}, \ldots, v_{n-1}\right)$ provide local coordinates on $X$ at a point $x \in f_{p} \backslash\left\langle p_{1}, \ldots, p_{n-1}\right\rangle$. Writing down the parametric equations for $X$ around $f_{p}$ we can easily get the matrix $M_{k}^{X}\left(t, v_{1}, \ldots, v_{n-1}\right)$ representing $j_{k}^{X}: V \rightarrow J_{k} L$ near $x$. Set

$$
\overline{M_{k-1}^{i}}=M_{\min \left\{k-1, r_{i}\right\}}^{i} .
$$

Lemma 1.1 [7, p. 152] We have

$$
M_{k}^{X}\left(t, v_{1}, \ldots, v_{n-1}\right)=\left(\begin{array}{ccccc}
\frac{v_{1} M_{k}^{1}}{M_{k-1}^{1}} & v_{2} M_{k}^{2} & \ldots & v_{n-1} M_{k}^{n-1} & M_{k}^{n} \\
0 & \frac{M_{k-1}^{2}}{2} & \ldots & 0 & 0 \\
\cdot & \cdot & \ldots & 0 & 0 \\
\cdot & \cdot & \ldots & \frac{\cdot}{M_{k-1}^{n-1}} & . \\
0 & 0 & \ldots & \cdot \\
0 & 0 & \ldots & 0 & 0
\end{array}\right)(t)
$$


Let $x \in f_{p} \backslash\left\langle p_{1}, \ldots, \widehat{p_{s}}, \ldots, p_{n}\right\rangle$, where $\widehat{ }$ denotes suppression. Up to reordering the $C_{i}$ 's, there is no restriction if we suppose that $s=n$, hence the matrix representing $j_{k}^{X}$ is that given by Lemma 1.1. Sometimes, however, it is convenient to order the $C_{i}$ 's according to some criterion (e. g., in such a way that $r_{1} \leq r_{2} \leq \cdots \leq r_{n}$ ). In this case, we can write $x=u_{1} p_{1}+\cdots+u_{s-1} p_{s-1}+p_{s}+u_{s} p_{s+1}+\cdots+u_{n-1} p_{n}$, and then, with respect to the local coordinates $\left(t, u_{1}, \ldots, u_{n-1}\right)$ the matrix representing $j_{k}^{X}$ near $x$ is the following

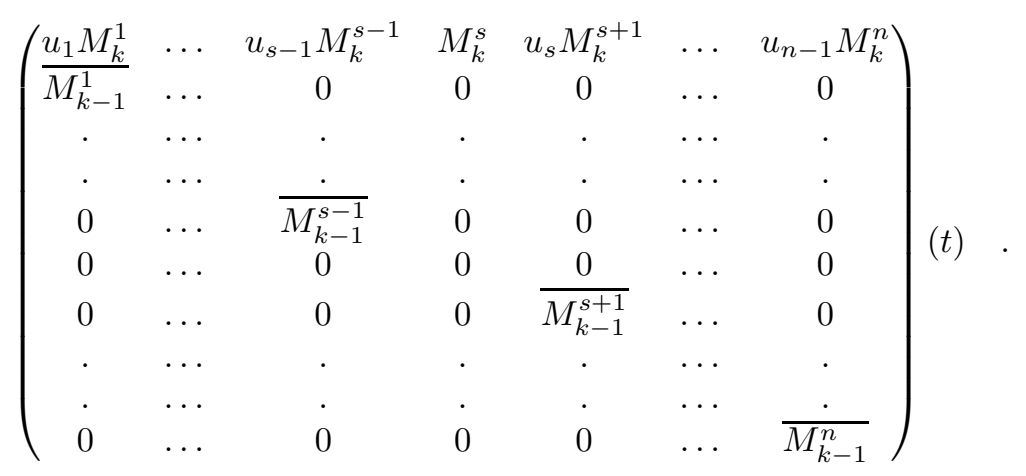

We say that two matrices $A$ and $B$ of type $m \times n$ are row equivalent if the vector subspace of $\mathbb{C}^{n}$ spanned by the rows of $A$ is the same as that spanned by the rows of $B$.

Here is an immediate application.

Theorem 1.2 Let $X$ be a decomposable scroll generated by $C_{1}, \ldots, C_{n}$ and let $\Phi_{2}(X)$ be its inflectional locus.

(1) The following three conditions are equivalent:

(i) $\left(f_{p} \backslash \bigcup_{i=1}^{n}\left\langle p_{1}, \ldots, \widehat{p}_{i}, \ldots, p_{n}\right\rangle\right) \cap \Phi_{2}(X) \neq \emptyset$;

(ii) $p_{i}$ is a flex of $C_{i}$ for every $i=1, \ldots, n$;

(iii) $f_{p} \subseteq \Phi_{2}(X)$.

(2) $p_{i} \in \Phi_{2}(X)$ if and only if it is a flex of $C_{i}$.

(3) Let $x \in \Phi_{2}(X)$ : if $x \in f_{p} \backslash\left\langle p_{1}, \ldots, \widehat{p_{s}}, \ldots, p_{n}\right\rangle$ then $p_{s}$ is a flex of $C_{s}$.

Pro of. To prove (1) it is enough to show that (i) $\Rightarrow$ (ii) $\Rightarrow$ (iii). Let $x \in f_{p} \backslash\left\langle p_{1}, \ldots, p_{n-1}\right\rangle$, so that we can write $x=v_{1} p_{1}+\ldots v_{n-1} p_{n-1}+p_{n}$. Then $x \in \Phi_{2}(X)$ if and only if $j_{2, x}^{X}: V \rightarrow\left(J_{2} L\right)_{x}$ has rank $<2 n+1$. Note that $\operatorname{rk}\left(\overline{M_{1}^{i}}(t)\right)=2$ and

$$
\operatorname{rk}\left(M_{2}^{i}(t)\right) \geq 2
$$

for every $i$ and for every $t$. Then Lemma 1.1 shows that $\operatorname{rk}\left(M_{2}^{X}\left(0, v_{1}, \ldots, v_{n-1}\right)\right)<2 n+1$ if and only if both $\operatorname{rk}\left(M_{2}^{n}(0)\right)=2$ and

$$
\operatorname{rk}\left(\left(v_{1} M_{2}^{1} \quad v_{2} M_{2}^{2} \quad \ldots \quad v_{n-1} M_{2}^{n-1}\right)(0)\right)=2 .
$$

The former condition says that $j_{2, p}: V_{n} \rightarrow\left(J_{2} \mathcal{L}_{n}\right)_{p}$ has rank 2 , while by (1) the latter one is equivalent to saying that either $v_{i}=0$ or $\operatorname{rk}\left(M_{2}^{i}(0)\right)=2$ for every $i=1, \ldots, n-1$. In conclusion we have that $j_{2, p}: V_{i} \rightarrow\left(J_{2} \mathcal{L}_{i}\right)_{p}$ has rank 2 for $i=n$ and for every $i$ such that $v_{i} \neq 0$. So, if $x \in \Phi_{2}(X)$ is a general point as in (i), we get (ii). On the other hand, if (ii) holds, then we see that $f_{p} \backslash\left\langle p_{1}, \ldots, p_{n-1}\right\rangle$, hence its closure $f_{p}$, lies in $\Phi_{2}(X)$. So (1) is proved. Moreover, the above argument proves the "only if" part of (2) when $x=p_{n}$, and (3) in the special case $s=n$. As to the "if" part of (2), note that if $x=p_{n}$ then the matrix $M_{2}^{X}(0,0, \ldots, 0)$ of Lemma 1.1 has the 
following special form:

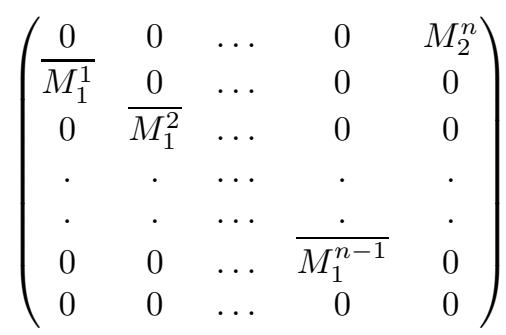

So, if $p_{n}$ is a flex of $C_{n}$ we get $\operatorname{rk}\left(M_{2}^{X}(0,0, \ldots, 0)\right)=2 n$, since $M_{2}^{n}(0)$ has rank 2 . Now, let $x$ be as in (3); so we can write $x=u_{1} p_{1}+\ldots u_{s-1} p_{s-1}+p_{s}+u_{s} p_{s+1}+\cdots+u_{n-1} p_{n}$. Then one can easily see that the matrix representing $j_{2}^{X}: V \rightarrow J_{2} L$ near $x$ is

$$
\left(\begin{array}{ccccccc}
u_{1} M_{2}^{1} & \ldots & u_{s-1} M_{2}^{s-1} & M_{2}^{s} & u_{s} M_{2}^{s+1} & \ldots & u_{n-1} M_{2}^{n} \\
M_{1}^{1} & \ldots & 0 & 0 & 0 & \ldots & 0 \\
\cdot & \ldots & . & . & . & \ldots & . \\
\cdot & \ldots & . & . & . & \ldots & \cdot \\
0 & \ldots & M_{1}^{s-1} & 0 & 0 & \ldots & 0 \\
0 & \ldots & 0 & 0 & 0 & \ldots & 0 \\
0 & \ldots & 0 & 0 & M_{1}^{s+1} & \ldots & 0 \\
. & \ldots & . & . & \cdot & \ldots & . \\
. & \ldots & . & . & . & \ldots & \frac{.}{M_{1}^{n}}
\end{array}\right)(t)
$$

Thus the same argument as above works and shows that since $x \in \Phi_{2}(X), p_{s}$ must be a flex of $C_{s}$. This completes the proof of (3) and (2).

Corollary $1.3 X$ is uninflected if and only if $C_{1}, \ldots, C_{n}$ are uninflected.

The same argument proving Theorem 1.2 says more.

Proposition 1.4 For any $x \in \Phi_{2}(X)$ we have

$$
\operatorname{Osc}_{x}^{2}(X)=\left\langle\operatorname{Osc}_{p_{1}}^{1}\left(C_{1}\right), \ldots, \operatorname{Osc}_{p_{s}}^{2}\left(C_{s}\right), \ldots, \operatorname{Osc}_{p_{n}}^{1}\left(C_{n}\right)\right\rangle
$$

for some $s$, where, $p_{s} \in \Phi_{2}\left(C_{s}\right)$. Moreover, $\operatorname{Osc}_{x}^{2}(X)$ is the same linear $\mathbb{P}^{2 n-1}$ for all $x \in \Phi_{2}(X) \cap f_{p}$.

Proof. First, suppose that $x \notin\left\langle p_{1}, \ldots, p_{n-1}\right\rangle$. Then $x=v_{1} p_{1}+\cdots+v_{n-1} p_{n-1}+p_{n}$. As $x \in \Phi_{2}(X)$, the first block of rows in the matrix $M_{2}^{X}\left(0, v_{1}, \ldots, v_{n-1}\right)$ appearing in Lemma 1.1 for $k=2$ has rank 2 . In particular, $p_{n} \in \Phi_{2}\left(C_{n}\right)$ by Theorem 1.2(3). Moreover, either $v_{i}=0$ or $v_{i} M_{2}^{i}$ is row equivalent to $\overline{M_{1}^{i}}$. Hence $M_{2}^{X}$ is row equivalent to the matrix

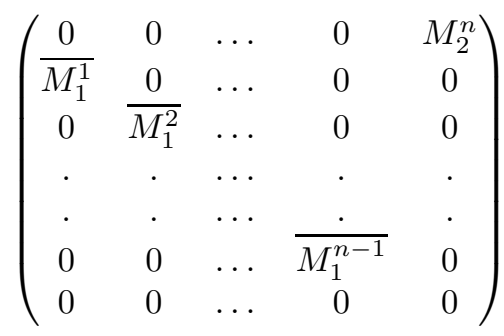

This means exactly that

$$
\operatorname{Osc}_{x}^{2}(X)=\left\langle\operatorname{Osc}_{p_{1}}^{1}\left(C_{1}\right), \ldots, \operatorname{Osc}_{p_{n-1}}^{1}\left(C_{n-1}\right), \operatorname{Osc}_{p_{n}}^{2}\left(C_{n}\right)\right\rangle
$$


Next, suppose that $x \in\left\langle p_{1}, \ldots, p_{n-1}\right\rangle \backslash\left\langle p_{1}, \ldots, p_{n-2}\right\rangle$. Then, $x=v_{1} p_{1}+\cdots+v_{n-2} p_{n-2}+p_{n-1}$ can also be written as $x=u_{1} p_{1}+\cdots+u_{s-1} p_{s-1}+p_{s}+u_{s} p_{s+1}+\cdots+u_{n-1} p_{n}$, as done after Lemma 1.1 , with $s=n-1$ and $u_{n-1}=0$. Then look at the matrix appearing after Lemma 1.1 in the present situation:

$$
\left(\begin{array}{ccccc}
u_{1} M_{2}^{1} & \ldots & u_{n-2} M_{2}^{n-2} & M_{2}^{n-1} & 0 \\
M_{1}^{1} & \ldots & 0 & 0 & 0 \\
\cdot & \ldots & . & . & \cdot \\
\cdot & \cdots & \frac{\cdot}{M_{1}^{n-2}} & 0 & 0 \\
0 & \ldots & 0 & 0 & \frac{0}{M_{1}^{n}}
\end{array}\right)(0)
$$

If $x \in \Phi_{2}(X)$, arguing as before we see that $p_{n-1} \in \Phi_{2}\left(C_{n-1}\right)$ and this matrix is row equivalent to

$$
\left(\begin{array}{ccccc}
\frac{0}{M_{1}^{1}} & \cdots & 0 & M_{2}^{n-1} & 0 \\
\cdot & \cdots & 0 & 0 & 0 \\
\cdot & \cdots & \frac{\cdot}{M_{1}^{n-2}} & 0 & 0 \\
0 & \cdots & . & \cdot \\
0 & \cdots & 0 & 0 & \frac{M_{1}^{n}}{}
\end{array}\right)(0) \quad .
$$

This means that

$$
\operatorname{Osc}_{x}^{2}(X)=\left\langle\operatorname{Osc}_{p_{1}}^{1}\left(C_{1}\right), \ldots, \operatorname{Osc}_{p_{n-2}}^{1}\left(C_{n-2}\right), \operatorname{Osc}_{p_{n-1}}^{2}\left(C_{n-1}\right), \operatorname{Osc}_{p_{n}}^{1}\left(C_{n}\right)\right\rangle
$$

Now, let $s \leq n-2$. By repeating the argument for $x \in\left\langle p_{1}, \ldots, p_{s}\right\rangle \backslash\left\langle p_{1}, \ldots, p_{s-1}\right\rangle$, we see that $p_{s} \in \Phi_{2}\left(C_{s}\right)$ and $\operatorname{Osc}_{x}^{2}(X)$ is the linear span of $\operatorname{Osc}_{p_{s}}^{2}\left(C_{s}\right)$ and the spaces $\operatorname{Osc}_{p_{i}}^{1}\left(C_{i}\right)$ for $i \neq s$. This proves the first assertion. Now, note that all $\operatorname{Osc}_{p_{i}}^{1}\left(C_{i}\right)$ are lines. Moreover, as we have shown, $p_{s}$ is a flex for $C_{s}$, hence $\operatorname{Osc}_{p_{s}}^{2}\left(C_{s}\right)$ is also a line. Thus, for any $x \in X, \operatorname{Osc}_{x}^{2}(X)$ is the linear space generated by the $n$ tangent lines to $C_{i}$ at $p_{i}$ for $i=1, \ldots, n$. Note that they generate a $\mathbb{P}^{2 n-1}$. It turns out that $\operatorname{Osc}_{x}^{2}(X)$ is the same $\mathbb{P}^{2 n-1}$ for all $x \in \Phi_{2}(X) \cap f_{p}$.

\section{Higher flexes and fibres}

Let $X$ be a decomposable scroll over a smooth curve $C$ generated by $C_{1}, \ldots, C_{n}$ as in Section 1 , and let $f_{p}=$ $\left\langle p_{1}, \ldots, p_{n}\right\rangle$ be the fibre over $p \in C$. In this section we explore some connections between the higher inflectional loci $\Phi_{k}(X)$ and the fibres of $X$.

Remark 2.1 We have

$$
\operatorname{Osc}_{p_{s}}^{k}(X)=\left\langle\operatorname{Osc}_{p_{1}}^{k-1}\left(C_{1}\right), \ldots, \operatorname{Osc}_{p_{s}}^{k}\left(C_{s}\right), \ldots, \operatorname{Osc}_{p_{n}}^{k-1}\left(C_{n}\right)\right\rangle
$$

for any $s=1, \ldots, n$ (the only $k$-th osculating space on the right hand is that at $\left.p_{s}\right)$. In particular, if $p_{s} \in \Phi_{k}\left(C_{s}\right)$, then $p_{s} \in \Phi_{k}(X)$.

Proof. Up to reordering the curves we can suppose that $s=n$. Then the matrix representing $j_{k, p_{s}}^{X}$ is, according to Lemma 1.1,

$$
\left(\begin{array}{cccc}
\frac{0}{M_{k-1}^{1}} & \cdots & 0 & M_{k}^{n} \\
\cdot & \cdots & 0 & 0 \\
\cdot & \cdots & \cdot & \cdot \\
0 & \cdots & \frac{\cdot}{M_{k-1}^{n-1}} & 0 \\
0 & \cdots & 0 & 0
\end{array}\right)
$$

This proves the first assertion. Note that all linear spaces appearing on the right hand of (2) are skew each other. 
Then the second assertion follows from the inequality:

$$
\begin{aligned}
\operatorname{dim}\left(\operatorname{Osc}_{p_{s}}^{k}(X)\right) & \leq(n-1)(k-1)+\operatorname{dim}\left(\operatorname{Osc}_{p_{s}}^{k}\left(C_{s}\right)\right)+(n-1) \\
& <(n-1) k+k=n k
\end{aligned}
$$

As to the converse, if $p_{s} \in \Phi_{k}(X)$, we cannot claim that $p_{s} \in \Phi_{k}\left(C_{s}\right)$ if $k>2$. However, we have

Remark 2.2 If $p_{s} \in \Phi_{k}(X)$, then either $p_{s} \in \Phi_{k}\left(C_{s}\right)$, or $p_{j} \in \Phi_{k-1}\left(C_{j}\right)$ for some $j \neq s$.

Proposition 2.3 Let $p_{i} \in \Phi_{k}\left(C_{i}\right)$ for $i=1, \ldots, \widehat{s}, \ldots, n$, where $\widehat{\wedge}$ denotes suppression. Then for every point $x \in f_{p} \backslash\left\langle p_{1}, \ldots, \widehat{p_{s}}, \ldots, p_{n}\right\rangle$ we have

$$
\operatorname{Osc}_{x}^{k}(X)=\left\langle\operatorname{Osc}_{p_{1}}^{k-1}\left(C_{1}\right), \ldots, \operatorname{Osc}_{p_{s}}^{k}\left(C_{s}\right), \ldots, \operatorname{Osc}_{p_{n}}^{k-1}\left(C_{n}\right)\right\rangle
$$

(the only $k$-th osculating space on the right hand is that at $p_{s}$ ).

Proof. Up to reordering we can suppose that $s=n$. Due to the assumption, we have $\operatorname{Osc}_{p_{i}}^{k}\left(C_{i}\right)=$ $\operatorname{Osc}_{p_{i}}^{k-1}\left(C_{i}\right)$ for $i=1, \ldots, n-1$. This means that the two matrices $M_{k}^{i}$ and $\overline{M_{k-1}^{i}}$ are row equivalent for $i=1, \ldots, n-1$. Now look at the matrix $M$ of Lemma 1.1. By subtracting suitable linear combinations of the subsequent rows from the first block of rows we see that $M$ is row equivalent to the matrix (3). This proves the assertion.

The same argument proves more. Actually, assume that $p_{i_{j}} \in \Phi_{k}\left(C_{i_{j}}\right)$ for $j=1, \ldots, s$ and set $\Lambda=$ $\left\langle p_{i_{1}}, \ldots, p_{i_{s}}\right\rangle$. Up to reordering we can suppose that $\left(i_{1}, \ldots, i_{s-1}, i_{s}\right)=(1, \ldots, s-1, n)$. Then for any $x \in \Lambda \backslash\left\langle p_{1}, \ldots, p_{s-1}\right\rangle$ we can write $x=v_{1} p_{1}+\ldots v_{s-1} p_{s-1}+p_{n}$. Arguing as in the proof of Proposition 2.3 we have that the matrices $M_{k}^{i}$ and $\overline{M_{k-1}^{i}}$ are row equivalent for $i=1, \ldots, s-1$. Now look at the matrix $M$ of Lemma 1.1, representing $j_{k, x}$. The first block of rows of $M$ is

$$
\left(\begin{array}{lllllll}
v_{1} M_{k}^{1} & \ldots & v_{s-1} M_{k}^{s-1} & 0 & \ldots & 0 & M_{k}^{n}
\end{array}\right) .
$$

By subtracting suitable linear combinations of the subsequent rows of $M$ from the first block we see that $M$ is row equivalent to the matrix in (3). Now, since also $p_{n} \in \Phi_{k}\left(C_{n}\right)$ we have $\operatorname{rk}\left(M_{k}^{n}\right)<k+1$ and then the same computation done to prove Remark 2.1 holds at $x$, giving $\operatorname{dim}\left(\operatorname{Osc}_{x}^{k}(X)\right)<n k$. Thus $\Lambda \backslash\left\langle p_{1}, \ldots, p_{s-1}\right\rangle \subseteq$ $\Phi_{k}(X)$. On the other hand $\Phi_{k}(X) \cap f_{p}$ is a Zariski closed subset, hence $\Lambda \subseteq \Phi_{k}(X)$.

Now suppose that $\left(i_{1}, \ldots, i_{s}\right)=(1, \ldots, s)$, with $s \leq n-1$ and $p_{n} \notin \Phi_{k}\left(C_{n}\right)$. Then $\operatorname{rk}\left(M_{k}^{n}\right)=k+1$, and the same argument as above applied to any point $x \in\left\langle p_{1}, \ldots p_{s}, p_{n}\right\rangle \backslash \Lambda$ shows that

$$
\operatorname{dim}\left(\operatorname{Osc}_{x}^{k}(X)\right)=\sum_{i=1}^{n-1} \operatorname{rk}\left(\overline{M_{k-1}^{i}}\right)+(k+1)-1 .
$$

In particular, if $p_{i} \notin \Phi_{k-1}\left(C_{i}\right)$ for $i=1, \ldots, s$, then all the first $n-1$ summands are equal to $k$, hence $\operatorname{dim}\left(\operatorname{Osc}_{x}^{k}(X)\right)=n k$, and so $x \notin \Phi_{k}(X)$. This proves the following

Proposition 2.4 If $p_{i_{j}} \in \Phi_{k}\left(C_{i_{j}}\right)$ for $j=1, \ldots, s \leq n$, then $\left\langle p_{i_{1}}, \ldots, p_{i_{s}}\right\rangle \subseteq \Phi_{k}(X)$. Moreover, if $p_{i_{j}} \in$ $\Phi_{k}\left(C_{i_{j}}\right) \backslash \Phi_{k-1}\left(C_{i_{j}}\right)$ for $j=1, \ldots, s \leq n-1$ and $p_{i_{j}} \notin \Phi_{k}\left(C_{i_{j}}\right)$ for $j=s+1, \ldots, n$, then $\Phi_{k}(X) \cap f_{p}=$ $\left\langle p_{i_{1}}, \ldots, p_{i_{s}}\right\rangle$.

Corollary 2.5 i) If $p_{i} \in \Phi_{k}\left(C_{i}\right)$ for every $i=1, \ldots, n$, then $f_{p} \subseteq \Phi_{k}(X)$.

ii) If $f_{p} \subseteq \Phi_{k}(X)$, then $p_{i} \in \Phi_{k}\left(C_{i}\right)$ for some $i$.

Proof. i) is obvious; ii) follows from Remark 2.2, taking into account the inclusion $\Phi_{k-1}\left(C_{j}\right) \subseteq \Phi_{k}\left(C_{j}\right)$

In particular, if $f_{p} \subseteq \Phi_{k}(X)$ and $k>2$, we see that not necessarily $p_{i} \in \Phi_{k}\left(C_{i}\right)$ for all $i$ 's. For $n=2$ we can be more explicit. 
Proposition 2.6 Let $n=2$ and $k \geq 2$. Then $f_{p} \subseteq \Phi_{k}(X)$ if and only if either

a) $p_{i} \in \Phi_{k-1}\left(C_{i}\right)$ for some $i$, or

b) $p_{i} \in \Phi_{k}\left(C_{i}\right)$ for $i=1,2$.

Pro of. Let $f_{p} \subseteq \Phi_{k}(X)$. By Corollary 2.5, ii), up to reordering, we can suppose that $p_{1} \in \Phi_{k}\left(C_{1}\right)$. Then, by Proposition 2.3, for every $x \in f_{p} \backslash\left\{p_{1}\right\}$, we have

$$
\operatorname{Osc}_{x}^{k}(X)=\left\langle\operatorname{Osc}_{p_{1}}^{k-1}\left(C_{1}\right), \operatorname{Osc}_{p_{2}}^{k}\left(C_{2}\right)\right\rangle
$$

Hence

$$
\operatorname{dim}\left(\operatorname{Osc}_{x}^{k}(X)\right)=\operatorname{dim}\left(\operatorname{Osc}_{p_{1}}^{k-1}\left(C_{1}\right)\right)+\operatorname{dim}\left(\operatorname{Osc}_{p_{2}}^{k}\left(C_{2}\right)\right)+1 .
$$

Since $x \in \Phi_{k}(X)$ this shows that either $p_{1} \in \Phi_{k-1}\left(C_{1}\right)$, case a), or $p_{2} \in \Phi_{k}\left(C_{2}\right)$, case b). To prove the converse, in both cases a) and b), up to renaming, we can assume that $p_{1} \in \Phi_{k}\left(C_{1}\right)$. Then Proposition 2.3 gives again (5) for any $x \in f_{p} \backslash\left\{p_{1}\right\}$ and then (6) shows that

$$
\operatorname{dim}\left(\operatorname{Osc}_{x}^{k}(X)\right) \leq\left\{\begin{array}{l}
k-2+k+1 \quad \text { in case a) } \\
k-1+(k-1)+1 \quad \text { in case } \mathbf{b}) .
\end{array}\right.
$$

Hence $f_{p} \backslash\left\{p_{1}\right\} \subseteq \Phi_{k}(X)$ in both cases, and then, taking the closure, we get $f_{p} \subseteq \Phi_{k}(X)$.

Theorem 2.7 Let $n=2$. Suppose that $x \in \Phi_{k}(X)$ and let $f_{p}$ be the fibre of $X$ containing $x$.

i) If $x \neq p_{1}, p_{2}$, then $f_{p} \subseteq \Phi_{k}(X)$;

ii) if $x=p_{i}$, then either $f_{p} \subseteq \Phi_{k}(X)$ or $p_{i} \in \Phi_{k}\left(C_{i}\right)$.

Proof. If $x \neq p_{1}$, then we can write $x=v p_{1}+p_{2}$. According to Lemma $1.1, j_{k, x}^{X}$ is represented by the following matrix

$$
M=\left(\begin{array}{cc}
\frac{v M_{k}^{1}}{M_{k-1}^{1}} & M_{k}^{2}
\end{array}\right)
$$

Since $x \in \Phi_{k}(X), M$ has rank $\leq 2 k$. This implies either

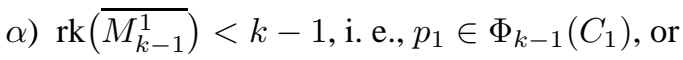

B) $\operatorname{rk}\left(v M_{k}^{1} M_{k}^{2}\right)<k$.

Condition $\beta$ ) in turn implies both $\operatorname{rk}\left(M_{k}^{1}\right)<k$ and $\operatorname{rk}\left(M_{k}^{2}\right)<k$. The latter condition means that $p_{2} \in \Phi_{k}\left(C_{2}\right)$, while the former is equivalent to saying that

$$
\text { either } v=0, \quad \text { or } \quad \operatorname{rk}\left(M_{k}^{1}\right)<k .
$$

In other words, either $x=p_{2}$ or $p_{1} \in \Phi_{k}\left(C_{1}\right)$. In conclusion, if $x \neq p_{1}, p_{2}$ then either

a) $p_{1} \in \Phi_{k-1}\left(C_{1}\right)$, or

в) $p_{i} \in \Phi_{k}\left(C_{i}\right)$ for $i=1,2$.

In both cases $f_{p} \subseteq \Phi_{k}(X)$ by Proposition 2.6. This proves i). Now let $x=p_{i}$. By Remark 2.2 either $p_{i} \in \Phi_{k}\left(C_{i}\right)$ or $p_{j} \in \Phi_{k-1}\left(C_{j}\right)$ for $j \neq i$. But in the latter case Proposition 2.6 says that $f_{p} \subseteq \Phi_{k}(X)$ again. This proves ii). 
Corollary 2.8 Let $n=2$. Then $\Phi_{k}(X)=\emptyset$ if and only if $\Phi_{k}\left(C_{i}\right)=\emptyset$ for $i=1,2$.

Proof. If $p_{i} \in \Phi_{k}\left(C_{i}\right)$ for some $i$, we know that $p_{i} \in \Phi_{k}(X)$ by Remark 2.2. This proves the "only if part". To prove the "if" part suppose, by contradiction, that $x \in \Phi_{k}(X)$, and let $f_{p}$ be the fibre of $X$ through $x$. By Theorem 2.7 either $f_{p} \subseteq \Phi_{k}(X)$ or $p_{i} \in \Phi_{k}\left(C_{i}\right)$ for some $i$. In both cases, taking into account Proposition 2.6, we see that $\Phi_{k}\left(C_{i}\right) \neq \emptyset$ for some $i$. But this is a contradiction.

Now suppose that $r_{1} \leq r_{2}$, where $\left\langle C_{i}\right\rangle=\mathbb{P}^{r_{i}}$. If $r_{1}<k-1$, then we have $\operatorname{dim}\left(\operatorname{Osc}_{y}^{k-1}\left(C_{1}\right)\right) \leq r_{1}<k-1$ for every $y \in C_{1}$. In other words, $\Phi_{k-1}\left(C_{1}\right)=C_{1}$ and therefore $\Phi_{k}(X)=X$ by Proposition 2.6. Let $r_{1} \geq k-1$. If $r_{2}<k$ (i. e., $r_{1}=r_{2}=k-1$ ), then $\Phi_{k}\left(C_{i}\right)=C_{i}$ for $i=1,2$, hence $\Phi_{k}(X)=X$ again, by Proposition 2.6. If $r_{1}=k-1$ but $r_{2} \geq k$ then $\Phi_{k}\left(C_{1}\right)=C_{1}$ but $\Phi_{k}\left(C_{2}\right) \subsetneq C_{2}$ (e. g., see [1, p. 37, Ex. C-2]); so $\Phi_{k}(X)$ contains every fibre of $X$ passing through a point of either $\Phi_{k-1}\left(C_{1}\right)$ or $\Phi_{k}\left(C_{2}\right)$. Taking into account also Remark 2.1, we thus get the following

Corollary 2.9 Let $n=2$ and suppose that $r_{1} \leq r_{2}$. If $\Phi_{k-1}\left(C_{1}\right)=\emptyset$ but $\Phi_{k}\left(C_{1}\right)=C_{1}$, then $\Phi_{k}(X)$ consists of $C_{1}$ plus the fibres containing a point of $\Phi_{k}\left(C_{2}\right)$.

\section{Surface scrolls: examples and applications}

In this section we focus on the surface case $(n=2)$. Let $X \subset \mathbb{P}^{N}$ be a decomposable surface scroll as in Section 1. We present some examples concerned with the dimension that $\operatorname{Osc}_{x}^{k}(X)$ can have at some point $x$, and with the structure of $\Phi_{2}(X)$, focusing in particular on the case of non-normal rational scrolls. First it is useful to recall the situation for normal rational scrolls.

Example 3.1 Notation as in Section 1; let $C=\mathbb{P}^{1}, \mathcal{E}=\mathcal{O}_{\mathbb{P}^{1}}\left(r_{1}\right) \oplus \mathcal{O}_{\mathbb{P}^{1}}\left(r_{2}\right)$, with $1 \leq r_{1} \leq r_{2}$, and let $X \subset \mathbb{P}^{N}$ be the image of $\mathbb{P}(\mathcal{E})$ in the embedding given by complete linear system associated with the tautological line bundle $L$. Note that $N=r_{1}+r_{2}+1$. Let $p=\left(t_{0}: t_{1}\right) \in \mathbb{P}^{1}$ and set $t=t_{1} / t_{0}$ (or $t_{0} / t_{1}$ ). At any point $x \in X \backslash C_{1}$ we can use local coordinates $(t, v)$ to write $x=v p_{1}+p_{2}$ on the fibre $f_{p}$; then, according to Lemma 1.1 , the homomorphism $j_{k}^{X}: H^{0}(X, L) \rightarrow J_{k} L$ is represented near $x$ by the matrix

$$
M_{k}^{X}(t, v)=\left(\begin{array}{cc}
\frac{v M_{k}^{1}}{M_{k-1}^{1}} & M_{k}^{2} \\
0 & 0
\end{array}\right)(t)
$$

Note that

$$
\operatorname{rk}\left(M_{k}^{2}(t)\right)=\min \left\{k+1, r_{2}+1\right\} .
$$

Moreover

$$
\operatorname{rk}\left(\overline{M_{k-1}^{1}}(t)\right)=\left\{\begin{array}{l}
\operatorname{rk}\left(M_{k-1}^{1}\right)=k \quad \text { if } k-1 \leq r_{1}, \\
\operatorname{rk}\left(M_{r_{1}}^{1}\right)=r_{1}+1 \quad \text { otherwise. }
\end{array}\right.
$$

It follows that

$$
\begin{aligned}
\operatorname{rk}\left(j_{k, x}^{X}\right) & =\operatorname{rk}\left(M_{k}^{X}(t, v)\right) \\
& =\operatorname{rk}\left(M_{k}^{2}(t)\right)+\operatorname{rk}\left(\overline{M_{k-1}^{1}}(t)\right) \\
& =\min \left\{k+1, r_{2}+1\right\}+\min \left\{k, r_{1}+1\right\} .
\end{aligned}
$$

Therefore

$$
\operatorname{dim}\left(\operatorname{Osc}_{x}^{k}(X)\right)= \begin{cases}2 k & \text { if } k \leq r_{1}+1 \\ k+r_{1}+1 & \text { if } r_{1}+1 \leq k \leq r_{2} \\ r_{1}+r_{2}+1 & \text { if } k \geq r_{2}\end{cases}
$$


Note that at any point $x \in X \backslash C_{1}$ the dimension of the $k$-th osculating space can be strictly smaller than $2 k$. This is obvious when $N<2 k$, but it can happen also for $k \leq\left[\frac{N-1}{2}\right]$, e. g., for a very unbalanced rational normal scroll (i. e., with invariant $e:=r_{2}-r_{1}$ very large). In fact, for $k \leq\left[\frac{N-1}{2}\right]$ we have $\operatorname{dim}\left(\operatorname{Osc}_{x}^{k}(X)\right)<2 k$ if $r_{1}+1<k$ from (8). This means $N+1-e<2 k \leq N-1$, hence $e \geq 3$ is enough. For $k \geq 2$, (8) also shows that

$$
k+2 \leq \operatorname{dim}\left(\operatorname{Osc}_{x}^{k}(X)\right) \leq 2 k
$$

at any point $x \in X \backslash C_{1}$. In particular, letting $k=2$ we see that $\operatorname{dim}\left(\operatorname{Osc}_{x}^{2}(X)\right)=4$ for any $x \in X \backslash C_{1}$.

We want to stress that $X$ was linearly normal in the example above. Here is an enlightening example showing how small the dimension of $\operatorname{Osc}_{x}^{k}(X)$ can be at some point $x$, for any $k$, when we drop linear normality.

Example 3.2 Fix integers $k \geq 2$ and $r=r_{2} \geq 3$. Let $C=\mathbb{P}^{1}, \mathcal{E}=\mathcal{L}_{1} \oplus \mathcal{L}_{2}$, where $\mathcal{L}_{1}=\mathcal{O}_{\mathbb{P}^{1}}(1)$, $\mathcal{L}_{2}=\mathcal{O}_{\mathbb{P}^{1}}(k+r-1)$, and let $V_{1}=H^{0}\left(\mathbb{P}^{1}, \mathcal{L}_{1}\right)=\left\langle t_{0}, t_{1}\right\rangle$,

$$
V_{2}=\left\langle t_{0}^{k+r-1}, t_{0}^{k+r-2} t_{1}, t_{0}^{r-2} t_{1}^{k+1}, \ldots, t_{0} t_{1}^{k+r-2}, t_{1}^{k+r-1}\right\rangle .
$$

Note that $\varphi_{2}: \mathbb{P}^{1} \rightarrow \mathbb{P}^{r}$ defines an embedding, which is not linearly normal, since $\operatorname{dim} V_{2}=r+1<h^{0}\left(\mathcal{L}_{2}\right)$. Then $X \subset \mathbb{P}^{r+2}$, defined as in Section 1, is a rational non-normal scroll. Let $L$ be the hyperplane bundle and let $V \subset H^{0}(X, L)$ be the subspace giving rise to the embedding. Note that at the point $p \in \mathbb{P}^{1}$, corresponding to $\left(t_{0}: t_{1}\right)=(1: 0)$ we have

$$
\left|V_{2}-2 p\right|=\cdots=\left|V_{2}-(k+1) p\right| .
$$

This means that for every $h,(2 \leq h \leq k)$ the homomorphism

$$
j_{h, p}^{C_{2}}: V_{2} \rightarrow\left(J_{k} \mathcal{L}_{2}\right)_{p}
$$

has a 2-dimensional image (isomorphic to $\left.\left(J_{1} \mathcal{L}_{2}\right)_{p}\right)$, i. e., $\operatorname{rk}\left(j_{k, p}^{C_{2}}\right)=2$. On the other hand, at every point $q \in \mathbb{P}^{1}$ it is obvious that $\operatorname{rk}\left(j_{h, q}^{C_{1}}\right)=2$ for any $h \geq 1$. Now, let $x \in f_{p}$. If $x \in f_{p} \backslash\left\{p_{2}\right\}$, Proposition 2.3 shows that

$$
\operatorname{Osc}_{x}^{h}(X)=\left\langle\operatorname{Osc}_{p_{1}}^{h}\left(C_{1}\right), \operatorname{Osc}_{p_{2}}^{h-1}\left(C_{2}\right)\right\rangle
$$

for any $h=2, \ldots, k$. On the other hand, Remark 2.1 tells us that

$$
\operatorname{Osc}_{p_{2}}^{h}(X)=\left\langle\operatorname{Osc}_{p_{1}}^{h-1}\left(C_{1}\right), \operatorname{Osc}_{p_{2}}^{h}\left(C_{2}\right)\right\rangle
$$

for any $h \geq 2$. In both cases $\operatorname{Osc}_{x}^{h}(X)$ is the linear span of two skew lines, namely $C_{1}$ and $\operatorname{Osc}_{p_{2}}^{1}\left(C_{2}\right)$; hence

$$
\operatorname{Osc}_{x}^{k}(X)=\operatorname{Osc}_{x}^{k-1}(X)=\cdots=\operatorname{Osc}_{x}^{2}(X) \text { for every } k \geq 3,
$$

at every point $x \in f_{p}$. In particular,

$$
\operatorname{dim}\left(\operatorname{Osc}_{x}^{k}(S)\right)=3 \quad \text { for all } k \geq 2 \text { at any point } x \in f_{p} .
$$

We recall that if $X \in \mathbb{P}^{N}$ is any scroll of dimension $n$, then $\operatorname{dim}\left(\operatorname{Osc}_{x}^{2}(X)\right) \geq n+1$ [2] (see also [6] for $n=2)$.

Example 3.3 Let $C=\mathbb{P}^{1}, \mathcal{L}_{1}=\mathcal{O}_{\mathbb{P}^{1}}(m)$ with $m \geq 2, V_{1}=H^{0}\left(\mathbb{P}^{1}, \mathcal{L}_{1}\right)$, and consider $\mathcal{L}_{2}=\mathcal{O}_{\mathbb{P}^{1}}(d)$ with $d \geq m+2$. The vector space $H^{0}\left(\mathbb{P}^{1}, \mathcal{L}_{2}\right)$ defines an embedding of $C$ as a rational normal curve $\Gamma \subset \mathbb{P}^{d}$. Projecting $\Gamma$ from a general linear space $T$ of dimension $d-m-2$ to a $\mathbb{P}^{m+1}$ we get an embedding. Let $V_{2}=V(T)$ be the vector subspace of $H^{0}\left(\mathbb{P}^{1}, \mathcal{L}_{2}\right)$ corresponding to this embedding. Let $C_{i}$ be the image of $C$ in the embedding defined by $V_{i}, i=1,2$, and in the space $\mathbb{P}^{2 m+2}=\mathbb{P}\left(V_{1} \oplus V_{2}\right)$ consider the decomposable rational scroll $X$ generated by $C_{1}$ and $C_{2}$. Note that $X=\mathbb{F}_{d-m}$. We claim that $\Phi_{m}(X)=\emptyset$. Of course $\Phi_{m}\left(C_{1}\right)=\emptyset$. Let $O_{\Gamma}$ be the $m$-th osculating developable of $\Gamma$ (i. e., the variety generated by the linear $\operatorname{spaces}^{\operatorname{Osc}_{x}^{m}}(\Gamma)$, as $x$ varies on $\Gamma)$. Note that $\operatorname{dim}\left(O_{\Gamma}\right)=m+1$, hence $T \cap O_{\Gamma}=\emptyset$ for a general $T$. Since no osculating space $\operatorname{Osc}_{x}^{m}(\Gamma)$ meets the center of projection $T$, we conclude that $\Phi_{m}\left(C_{2}\right)=\emptyset$. Then the claim follows from Corollary 2.8. 
Let us recall the following conjecture of Piene-Tai [10]. Let $S \subset \mathbb{P}^{N}(N \geq 5)$ be a non-degenerate smooth projective surface such that $\operatorname{dim}\left(\operatorname{Osc}_{x}^{k}(S)\right) \leq 2 k$ for all points $x \in S$ and for every $k$, with equality for $k=$ $\left[\frac{N-1}{2}\right]$, where [ ] stands for the greatest integer function.

(i) If $N$ is odd, then $S$ is the balanced rational normal scroll of degree $N-1$ (i. e., $S$ is $\mathbb{F}_{0}$ embedded by $\left.\left|C_{0}+\left[\frac{N-1}{2}\right] f\right|\right)$.

(ii) If $N$ is even, then $S$ is the semibalanced rational normal scroll of degree $N-1$ (i. e., $S$ is $\mathbb{F}_{1}$ embedded by $\left.\left|C_{0}+\left(\left[\frac{N-1}{2}\right]+1\right) f\right|\right)$.

Part (i) of this conjecture is true, as proved in [3], while part (ii) is not (see [6, Theorem A and comment after Corollary 2.3]). Example 3.3 provides a new series of counterexamples to the even dimensional part of the conjecture. We want to stress that all these scrolls are decomposable, while those appearing in [6, Theorem A] are not, all being isomorphic to the elliptic $\mathbb{P}^{1}$-bundle of invariant -1 . Moreover, we have the following characterization, which provides more information in order to correct the conjecture.

Theorem 3.4 Let $X \subset \mathbb{P}^{2 m+2}(m \geq 2)$ be a decomposable scroll with $n=2$ such that $\Phi_{m}(X)=\emptyset$. Then either $X$ is the semibalanced rational normal scroll of degree $m+1$, or $X$ is of the type described in Example 3.3 .

Proof. By Corollary 2.8 it must be $\Phi_{m}\left(C_{i}\right)=\emptyset$, for $i=1,2$. In particular, $C_{1}$ cannot be a line, hence $r_{1}=\operatorname{dim}\left(\left\langle C_{1}\right\rangle\right) \geq 2$. We can assume that $r_{1} \leq r_{2}$ and then from $r_{1}+r_{2}+1=2 m+2$ we get that $r_{1} \leq m$. As $\Phi_{m}\left(C_{1}\right)=\emptyset$, this implies that $r_{1}=m$, and then $r_{2}=m+1$. So $C_{1}$ is a rational normal curve of degree $m$ in $\mathbb{P}^{m}$ while $C_{2}$ is either the rational normal curve of degree $m+1$ in $\mathbb{P}^{m+1}$ or any other rational non-normal curve of some degree $d \geq m+2$ in $\mathbb{P}^{m+1}$. In the former case $X$ is the semibalanced rational normal scroll. In the latter, $C_{2}$ is obtained by projecting a rational normal curve of degree $d$ in $\mathbb{P}^{d}$ to $\mathbb{P}^{m+1}$ from a general center as in Example 3.3.

The examples in the next part of this section are concerned with $\Phi_{2}(X)$. First we would like to stress that for the cubic scroll $X \subset \mathbb{P}^{4}$ the inflectional locus $\Phi_{2}(X)$ consists exactly of the generating line $C_{1}$. In fact this is the only semi-balanced rational normal scroll which is not uninflected. As to quartic rational normal scrolls in $\mathbb{P}^{5}$ the situation is also well known [11]. Let us note that the one isomorphic to $\mathbb{F}_{0}$ is uninflected according to Corollary 1.3 , being generated by two conics $C_{1}, C_{2}$. On the other hand, the one isomorphic to $\mathbb{F}_{2}$ is generated by a line $C_{1}$ and a rational normal cubic $C_{2}$, which has no flexes. Hence, according to Theorem 1.2(1) its inflectional locus $\Phi_{2}$ consists exactly of $C_{1}$.

Example 3.5 We consider quintic non-normal rational scrolls in $\mathbb{P}^{5}$. Let $X$ be as in Example 3.3, with $m=1$ and $d=4$. According to Theorem 1.2(1), the inflectional locus $\Phi_{2}(X)$ consists of the line $C_{1}$ and the fibres passing through the flexes of the non-normal quartic rational curve $C_{2}$. Now the center of projection $T$ is a point. If $T \notin O_{\Gamma}$, then $C_{2}$ has no flexes, as we said, and then $\Phi_{2}(X)=C_{1}$. On the other hand, if $c \in O_{\Gamma}$, then $C_{2}$ has $\epsilon$ flexes, where $\epsilon$ is the number of osculating planes to $\Gamma$ passing through $T$. According to the enumerative formula counting the weighted number of 2-osculating lines and 3-osculating planes to $C_{2}$ [8, Theorem 3.2] we can see that $\epsilon=1$ or 2. Depending on this, $\Phi_{2}(X)$ consists of $C_{1}$ plus one or two fibres.

Example 3.6 In the same vein we can construct non-normal rational scrolls having a finite inflectional locus. Let $C, \mathcal{L}_{2}, V_{2}$ be as in the previous example but now put $\mathcal{L}_{1}=\mathcal{O}_{\mathbb{P}^{1}}(2)$ and $V_{1}=H^{0}\left(\mathbb{P}^{1}, \mathcal{L}_{1}\right)$. Again let $C_{i}$ be the image of $C$ in the embedding defined by $V_{i}, i=1,2$, and in the space $\mathbb{P}^{6}=\mathbb{P}\left(V_{1} \oplus V_{2}\right)$ consider the decomposable sextic rational scroll $X$ generated by $C_{1}$ and $C_{2}$. Now $C_{1}$ is a conic, hence it has no flexes. On the other hand $C_{2}$ has $\epsilon=1$ or 2 flexes provided that the projection of $\Gamma$ giving rise to $C_{2}$ is made from a center $T \in O_{\Gamma}$. Therefore, according to Theorem $1.2\left((1)\right.$ and (2)), $\Phi_{2}(X)$ consists of one or two points (the flexes of $C_{2}$ ).

Example 3.7 Let $C$ be a smooth curve of genus 1, $\mathcal{L}_{1}=\mathcal{O}_{C}(3 p)$, for some point $p \in C, V_{1}=H^{0}\left(C, \mathcal{L}_{1}\right)$. Then $C_{1}=\varphi_{1}(C)$ is a smooth plane cubic having exactly 9 flexes, one of which is $p_{1}:=\varphi_{1}(p)$. Now let $\mathcal{L}_{2}$ be a line bundle of degree 5 on $C$. The vector space $H^{0}\left(C, \mathcal{L}_{2}\right)$ defines an embedding of $C$ in $\mathbb{P}^{4}$ whose image, say $\Gamma$, is a quintic normal elliptic curve: then $\Gamma$ has no flexes (but 25 hyperflexes). Projecting $\Gamma$ from a point $c \in \mathbb{P}^{4} \backslash \operatorname{Sec}(\Gamma)$ to a $\mathbb{P}^{3}$ we get an embedding; let $V_{2}=V(c)$ be the corresponding vector subspace of $H^{0}\left(C, \mathcal{L}_{2}\right)$ 
and let $C_{2}$ be the image of $C$ in the embedding $\varphi_{2}: C \rightarrow \mathbb{P}^{3}$ defined by $V_{2}$. In the space $\mathbb{P}^{6}=\mathbb{P}\left(V_{1} \oplus V_{2}\right)$ consider the decomposable elliptic scroll $X$ generated by $C_{1}$ and $C_{2}$. It can happen that $p_{2}:=\varphi_{2}(p)$ is a flex of $C_{2}$ or not. According to Theorem 1.2(1), in the former case the whole fibre $f_{p}$ is in the inflectional locus $\Phi_{2}(X)$, while in the latter we have $\Phi_{2}(X) \cap f_{p}=\left\{p_{1}\right\}$. Note that if $c$ is general enough, then $C_{2}$ has no flexes and therefore $X$ has only 9 flexes: those of $C_{1}$.

\section{The second discriminant locus of decomposable scrolls}

Let $(X, L, W)$ be as at the beginning of Section 0 , and let $\mathcal{U} \subseteq X$ be the Zariski dense open subset of $X$ where $j_{k, x}^{(X, W)}: W \rightarrow\left(J_{k} L\right)_{x}$ attains the maximum rank $s(k)+1$. If $x \in \mathcal{U}$, the fact that $H \in|W|$ is a $k$-th osculating hyperplane to $X$ at $x$ is equivalent to the fact that $H=(\sigma)_{0}$, where $\sigma \in W$ and $j_{k, x}(\sigma)=0$. Equivalently, this means that $H \in|W-(k+1) x|$, i. e., the hyperplane section cut out by $H$ on $X$ has a point of multiplicity $\geq(k+1)$ at $x$. Note however that if $x \notin \mathcal{U}$ and $H \in \mid W-(k+1) x) \mid$, this does not necessarily mean that $H \in X_{k}^{\vee}$. Actually $H \in X_{k}^{\vee}$ if and only if $H$ is a limit of $k$-th osculating hyperplanes to $X$ at points of $\mathcal{U}$. On the other hand we can consider the $k$-th discriminant locus $\mathcal{D}_{k}(X, W)$ of $(X, W)$, which is defined as the image of

$$
\mathcal{J}:=\{(x, H) \in X \times|W||H \in| W-(k+1) x \mid\}
$$

via the second projection of $X \times|W|$. It parameterizes all hyperplane sections of $X \subset \mathbb{P}^{N}$ admitting a singular point of multiplicity $\geq k+1$; of course $\mathcal{D}_{k}(X, W) \supseteq X_{k}^{\vee}$ with equality if and only if $\mathcal{U}=X$, i. e., if and only if

$$
\operatorname{dim}\left(\operatorname{Osc}_{x}^{k}(X)\right)=s(k) \quad \text { for every } x \in X .
$$

In general $\mathcal{D}_{k}(X, W)$ contains some extra components coming from the irreducible components of $\Phi_{k}(X)$.

The discussion above says that $\mathcal{D}_{k}(X, W)=X_{k}^{\vee}$ if and only if $\Phi_{k}(X)=\emptyset$. From this point of view, the characterization of balanced rational normal surface scrolls due to Ballico, Piene and Tai [3], mentioned after Example 3.3, can be rephrased as follows.

Proposition 4.1 Let $X \subset \mathbb{P}^{N}$ be any smooth surface, where $N=2 m+1 \geq 5$. Then $\mathcal{D}_{m}(X, W)=X_{m}^{\vee}$ if and only if $X=\mathbb{P}^{1} \times \mathbb{P}^{1}$ and $W=H^{0}\left(\mathbb{P}^{1} \times \mathbb{P}^{1}, \mathcal{O}_{\mathbb{P}^{1} \times \mathbb{P}^{1}}(1, m)\right)$.

Now, let $X \subset \mathbb{P}^{N}=\mathbb{P}(V)$ be a decomposable scroll as in Section 1. For simplicity we identify $X$ with the corresponding abstract projective bundle $P$. So, we denote by $\mathcal{D}_{2}(X, V)$ the second discriminant locus of $(P, L, V)$. Its main component is the second dual variety $X_{2}^{\vee}$ of $X$. Note that if $X$ is not linearly normal then $X_{2}^{\vee}$ corresponds to a suitable linear section of the second dual variety of the linearly normal scroll giving rise to $X$ via the projection to $\mathbb{P}^{N}$. Here, relying on the results of Sections 1 and 2, we want to describe the extra components of $\mathcal{D}_{2}(X, V)$. Of course we assume that $\Phi_{2}(X) \neq \emptyset$. As a first thing we need to describe the irreducible components of $\Phi_{2}(X)$.

Proposition 4.2 Let $X \subset \mathbb{P}^{N}$ be a decomposable scroll as in Section 1 , generated by $C_{1}, \ldots, C_{n}$, and assume that $\Phi_{2}(X) \neq X$. Let $G$ be an irreducible component of $\Phi_{2}(X)$. Then, up to reordering the curves $C_{i}$ 's, either

(1) $G=\left\langle p_{1}, \ldots, p_{s}\right\rangle \subseteq f_{p}=\left\langle p_{1}, \ldots, p_{n}\right\rangle$, or

(2) $X$ is rational and $G=C_{1} \times\left\langle p_{1}, \ldots, p_{s}\right\rangle$ is the image of $\mathbb{P}^{1} \times \mathbb{P}^{s-1}$ via the Segre embedding.

Moreover,

$$
\operatorname{Osc}_{x}^{2}(X)=\left\langle\operatorname{Osc}_{p_{1}}^{2}\left(C_{1}\right), \operatorname{Osc}_{p_{2}}^{1}\left(C_{2}\right), \ldots, \operatorname{Osc}_{p_{n}}^{1}\left(C_{n}\right)\right\rangle
$$

for all $x \in G$ in case (1) and for all $x \in G \cap f_{p}$ in case (2). In particular, $\operatorname{dim}\left(\operatorname{Osc}_{x}^{2}(X)\right)=2 n-1$ for any $x \in G$ in both cases.

Proof. As $\Phi_{2}(X) \neq \emptyset$ it follows from Theorem 1.2(3) that $\Phi_{2}\left(C_{i}\right) \neq \emptyset$ for some $i$. If $\Phi_{2}\left(C_{i}\right) \neq C_{i}$ for every $i=1, \ldots, n$, then we get an irreducible component as in case (1). Actually, up to reordering the curves, we can assume that $p_{i} \in \Phi_{2}\left(C_{i}\right)$ for $i=1, \ldots, s$. Then $G:=\left\langle p_{1}, \ldots, p_{s}\right\rangle \subseteq \Phi_{2}(X)$ by Proposition 2.4. Moreover, 
since $\Phi_{2}\left(C_{i}\right)$ is a finite set for every $i$, we conclude that $G$ is an irreducible component of $\Phi_{2}(X)$. Now suppose that $\Phi_{2}\left(C_{i}\right)=C_{i}$ for some $i$. Then up to reordering the curves we can assume that $\Phi_{2}\left(C_{i}\right)=C_{i}$ for $i=1, \ldots, s$ and $\Phi_{2}\left(C_{i}\right) \neq C_{i}$ for $i>s$. This implies that $C_{i}$ is a line for $i=1, \ldots, s$ and a rational curve of higher degree for $i>s$. In particular $C=\mathbb{P}^{1}$, i. e., $X$ is a rational scroll. Moreover $G_{p}:=\left\langle p_{1}, \ldots, p_{s}\right\rangle \subseteq \Phi_{2}(X)$ by Proposition 2.4 , for every $p \in \mathbb{P}^{1}$. Let $G:=\bigcup_{p \in \mathbb{P}^{1}} G_{p}$. Then $G$ is the sub-scroll of $X$ generated by the lines $C_{1}, \ldots C_{s}$. In other words, $G$ is $\mathbb{P}\left(\mathcal{O}_{\mathbb{P}^{1}}(1)^{\oplus s}\right)=\mathbb{P}^{1} \times \mathbb{P}^{s-1}$ embedded in the linear span of $C_{1}, \ldots, C_{s}$ via the Segre embedding. This gives case (2) and there are no further possibilities. The last assertions follow from Proposition 1.4, since $G \subseteq \Phi_{2}(X)$.

Of course it may happen that a fibre $G_{p}$ of an irreducible component of $\Phi_{2}(X)$ of type (2) is contained in a larger component of $\Phi_{2}(X)$ of type (1). This happens if $C_{j}$ has a flex at the point $p_{j}$ for some $j>s$.

Now let us consider the second discriminant locus: for simplicity we set $\mathcal{D}=\mathcal{D}_{2}(X, V)$ and denote by $\mathcal{D}_{G}$ the component of $\mathcal{D}$ arising from an irreducible component $G$ of $\Phi_{2}(X)$. Then

$$
\mathcal{D}_{G}=\left\{H \in \mathbb{P}^{N \vee} \mid H \supseteq \operatorname{Osc}_{x}^{2}(X) \text { for any } x \in G\right\} .
$$

Let $G$ be an irreducible component of $\Phi_{2}(X)$. We say that $G$ is of type (1) or (2) according to the cases of Proposition 4.2. Let $G$ be of type (1). Then, recalling that $\operatorname{Osc}_{x}^{2}(X)$ is a fixed $\mathbb{P}^{2 n-1}$ for all $x \in G$, we conclude that the component $\mathcal{D}_{G}$ is a linear $\mathbb{P}^{N-2 n}$. Now suppose that $G$ is of type (2). By Proposition 4.2, Osc ${ }_{x}^{2}(X)$ is a fixed linear space $T_{p}:=\mathbb{P}^{2 n-1}$ for $x \in G \cap f_{p}$. So, letting $p$ vary on $\mathbb{P}^{1}$ we see that

$$
\mathcal{D}_{G}=\bigcup_{p \in \mathbb{P}^{1}}\left\{H \in \mathbb{P}^{N \vee} \mid H \supseteq T_{p}\right\} .
$$

We can think of $T_{p}$ as $\operatorname{Osc}_{p_{1}}^{2}(X)$. Note that if $\Phi_{2}(X) \neq X$, the tangent line to $C_{n}$ varies as $p$ varies on $C$. Hence for points $x, y \in G$, lying on general distinct fibres $f_{p}, f_{q}$ of $X$ we have

$$
\operatorname{Osc}_{x}^{2}(X)=\operatorname{Osc}_{p_{1}}^{2}(X) \neq \operatorname{Osc}_{q_{1}}^{2}(X)=\operatorname{Osc}_{y}^{2}(X)
$$

It follows that $\operatorname{dim}\left(\mathcal{D}_{G}\right)=N-2 n+1, \mathcal{D}_{G}$ being a family of $\mathbb{P}^{N-2 n}$ parameterized by $\mathbb{P}^{1}$. More precisely, we can describe the structure of $\mathcal{D}_{G}$ in this way. Consider the incidence correspondence

$$
\mathcal{P}=\left\{\left(p_{1}, H\right) \in C_{1} \times \mathbb{P}^{N \vee} \mid H \supseteq \operatorname{Osc}_{p_{1}}^{2}(X)\right\}
$$

Note that $\mathcal{P}$ is a $\mathbb{P}^{N-2 n}$-bundle over $C_{1}=\mathbb{P}^{1}$ via the first projection of $C_{1} \times \mathbb{P}^{N \vee}$, since $\operatorname{Osc}_{p_{1}}^{2}(X)$ is a $\mathbb{P}^{2 n-1}$ for any $p_{1} \in C_{1}$. Then $\mathcal{D}_{G}=\pi(\mathcal{P})$, where $\pi$ is the second projection of $C_{1} \times \mathbb{P}^{N \vee}$.

Example 4.3 Let $X$ be a decomposable scroll as in Section 1, generated by lines $C_{1}, \ldots, C_{n-1}$ and by a nondegenerate rational curve $C_{n} \subset \mathbb{P}^{r}, r=r_{n} \geq 3$, of degree $d$. Then $d \geq 3$ and $X \subset \mathbb{P}^{N}$, where $N=2 n-2+r$. According to Proposition 4.2, $G=C_{1} \times \mathbb{P}^{n-2}$, Segre embedded in $\mathbb{P}^{2 n-3}=\left\langle C_{1}, \ldots C_{n-1}\right\rangle$. Let $p, q$ be any two distinct points of $C=\mathbb{P}^{1}$. The tangent lines to $C_{n}$ at $p_{n}$ and $q_{n}$ generate at most a $\mathbb{P}^{3}$. Therefore

$$
\operatorname{dim}\left(\left\langle C_{1}, \ldots, C_{n-1}, \operatorname{Osc}_{p_{n}}^{1}\left(C_{n}\right), \operatorname{Osc}_{q_{n}}^{1}\left(C_{n}\right)\right\rangle\right) \leq 2 n+1 .
$$

Recall that the linear space above is just the linear $\operatorname{span}\left\langle\operatorname{Osc}_{p_{1}}^{2}(X), \operatorname{Osc}_{q_{1}}^{2}(X)\right\rangle$ by Proposition 4.2. So, if $r \geq 4$, for any two distinct points $p, q \in C$ there exists a hyperplane $H$ of $\mathbb{P}^{N}$ containing both $\operatorname{Osc}_{p_{1}}^{2}(X)$ and Osc $q_{1}^{2}(X)$. Note that any such a hyperplane corresponds to a singular point of $\mathcal{D}_{G}$ : actually, $\left.\pi\right|_{\mathcal{P}} ^{-1}(H)=\left\{\left(p_{1}, H\right),\left(q_{1}, H\right)\right\}$. In particular, if $r \geq 4$, then $\operatorname{Sing}\left(\mathcal{D}_{G}\right)$ contains the $\mathbb{P}^{2}$ parameterizing the double symmetric product of $C_{n}$ with itself. Now let $r=3$. If $d \geq 4$, then $C_{n}$ is not normal. Hence it is the image of a rational normal curve $\bar{C} \subset \mathbb{P}^{d}$ of degree $d$ via a projection from a general linear space $T$ of dimension $d-4$. Any two tangents to $\bar{C}$ span a $\mathbb{P}^{3}$, so, one sees by a dimension count that in the dual space $\mathbb{P}^{d \vee}$ there is a one dimensional family of hyperplanes of $\mathbb{P}^{d}$ containing two tangent lines to $\bar{C}$ and $T$. Projecting to $\mathbb{P}^{3}$ they provide infinitely many pairs of coplanar tangent lines to $C_{n}$ (see also [5, Remark 5.2]). This being a closed condition, implies that for any $p_{n} \in C_{n}$ there exists some other point $q_{n} \in C_{n}$ such that the two tangent lines $\operatorname{Osc}_{p_{n}}^{1}\left(C_{n}\right)$ and $\operatorname{Osc}_{q_{n}}^{1}\left(C_{n}\right)$ are coplanar. For such a pair of points,

$$
\operatorname{dim}\left(\left\langle C_{1}, \ldots, C_{n-1}, \operatorname{Osc}_{p_{n}}^{1}\left(C_{n}\right), \operatorname{Osc}_{q_{n}}^{1}\left(C_{n}\right)\right\rangle\right)=2 n .
$$


Hence $H:=\left\langle\operatorname{Osc}_{p_{1}}^{2}(X), \operatorname{Osc}_{q_{1}}^{2}(X)\right\rangle$ is a hyperplane of $\mathbb{P}^{2 n+1}$ giving rise to a singular point of $\mathcal{D}_{G}$. Finally, let $r=3=d$. Then $C_{n}$ is a twisted cubic. Being a rational normal curve, we know that any two distinct tangent lines to $C_{n}$ do not meet. Thus, for any two distinct points $p, q \in C$ we have that

$$
\left\langle\operatorname{Osc}_{p_{1}}^{2}(X), \operatorname{Osc}_{q_{1}}^{2}(X)\right\rangle=\left\langle C_{1}, \ldots, C_{n-1}, \operatorname{Osc}_{p_{n}}^{1}\left(C_{n}\right), \operatorname{Osc}_{q_{n}}^{1}\left(C_{n}\right)\right\rangle=\left\langle C_{1}, \ldots, C_{n}\right\rangle
$$

is the whole $\mathbb{P}^{2 n+1}$. In fact, in this case $\mathcal{D}_{G}$ is a scroll over $C$ (see Proposition 4.4 below).

What we said in Example 4.3 when either $r \geq 4$ or $r=3$ and $d \geq 4$ holds, "a fortiori", if $C_{1}, \ldots, C_{s}$ are lines $(s \geq 1)$ and for some $i=s+1, \ldots, n$ either $r_{i} \geq 4$ or $r_{i}=3$ and $\operatorname{deg} C_{i} \geq 4$. Actually, also in this case there are hyperplanes $H$ of $\mathbb{P}^{N}$ containing both $\operatorname{Osc}_{p_{1}}^{2}(X)$ and $\operatorname{Osc}_{q_{1}}^{2}(X)$, for distinct points $p, q$ of $C$, and any such hyperplane gives rise to a singular point of $\mathcal{D}_{G}$. From now on in this Section, we assume that

$$
1=\operatorname{deg} C_{1}=\cdots=\operatorname{deg} C_{s}<\operatorname{deg} C_{s+1} \leq \cdots \leq \operatorname{deg} C_{n} .
$$

Example 4.3 shows that $\mathcal{D}_{G}$ is not a scroll if $\operatorname{deg} C_{n} \geq 4$. On the other hand, we can prove the following

Proposition 4.4 Let $X$ be a decomposable scroll generated by $C_{1}, \ldots, C_{n}$, where $C_{i}$ is a line for $i=1, \ldots, s$ and

$$
2 \leq \operatorname{deg} C_{s+1} \leq \cdots \leq \operatorname{deg} C_{n} \leq 3 .
$$

Let $G$ be the sub-scroll of $X$ generated by $C_{1}, \ldots, C_{s}$. Then $\mathcal{D}_{G}$ is a rational scroll.

We need to point out some facts.

Remark 4.5 Let $Y$ be the decomposable scroll generated by $C_{s+1}, \ldots, C_{n}$, and let $\mathbb{P}^{M}$ be its linear span in $\mathbb{P}^{N}$. We denote by $\Sigma$ the minimal sub-scroll of $Y$ generated by the curves $C_{s+1}, \ldots, C_{n-1}$ and by $F$ any fibre of $Y$. Note that $\Sigma \cap C_{n}=\emptyset$, while $\Sigma \cap F$ is a hyperplane of $F$. We have $\operatorname{Pic}(Y) \cong \mathbb{Z}^{2}$ and we can choose as generators the classes of $\Sigma$ and $F$. Then:

(i) any hyperplane of $\mathbb{P}^{M}$ cuts $Y$ along a divisor $D$ linearly equivalent to $\Sigma+b F$, for some integer $b>0$. In particular, since $\Sigma$ does not meet $C_{n}$ we see that

$$
\operatorname{deg} C_{n}=D C_{n}=(\Sigma+b F) C_{n}=b .
$$

(ii) For any hyperplane $H$ of $\mathbb{P}^{N}$ not containing $\mathbb{P}^{M}$ set $h:=H \cap \mathbb{P}^{M}$. If $h$ contains $\operatorname{Osc}_{p_{i}}^{1}\left(C_{i}\right)$ for every $i=s+1, \ldots, n$ then $h$ cuts $Y$ along a divisor of the form $D=2 F_{p}+R$ where $F_{p}=\left\langle p_{s+1}, \ldots, p_{n}\right\rangle$ and $R$ is an effective divisor linearly equivalent to $\Sigma+\beta F$, with $\beta \geq 0$. Indeed, the tangent space to $Y$ at $p_{i}$ is

$$
\operatorname{Osc}_{p_{i}}^{1}(Y)=\left\langle p_{s+1}, \ldots, \operatorname{Osc}_{p_{i}}^{1}\left(C_{i}\right), \ldots, p_{n}\right\rangle
$$

for $i=s+1, \ldots, n$, by Remark 2.1 with $k=1$. Hence $h$ is tangent to $Y$ at all points $p_{s+1}, \ldots, p_{n}$. Since they are linearly independent, this says that $h$ is tangent to $Y$ along the whole fibre $F_{p}$. Thus the divisor $D$ cut out by $h$ on $Y$ is singular at all points of $F_{p}$, hence the summand $2 F_{p}$ appears in the expression of $D$ as positive linear combination of its irreducible components.

Now we can prove Proposition 4.4.

Pro of. As the fibres of $\mathcal{P}$ are mapped linearly into $\mathbb{P}^{N \vee}$ by $\pi$, it is enough to show that the bundle projection of $\mathcal{P}$ induces a morphism $\mathcal{D}_{G} \rightarrow C_{1}$. To do that we prove that $\pi$ is bijective, i. e., for any $H \in \mathcal{D}_{G}$, the fibre $\left.\pi\right|_{\mathcal{P}} ^{-1}(H)$ consist of a single element. Equivalently, for any pair of distinct points $p, q \in C$, there is no hyperplane $H \subset \mathbb{P}^{N}$ containing both $\operatorname{Osc}_{p_{1}}^{2}(X)$ and $\operatorname{Osc}_{q_{1}}^{2}(X)$. Set

$$
R_{p}=\left\langle\operatorname{Osc}_{p_{s+1}}^{1}\left(C_{s+1}\right), \ldots, \operatorname{Osc}_{p_{n}}^{1}\left(C_{n}\right)\right\rangle, \quad R_{q}=\left\langle\operatorname{Osc}_{q_{s+1}}^{1}\left(C_{s+1}\right), \ldots, \operatorname{Osc}_{q_{n}}^{1}\left(C_{n}\right)\right\rangle
$$

By Proposition 4.2 we know that

$$
\operatorname{Osc}_{p_{1}}^{2}(X)=\left\langle C_{1}, \ldots, C_{s}, R_{p}\right\rangle, \quad \operatorname{Osc}_{q_{1}}^{2}(X)=\left\langle C_{1}, \ldots, C_{s}, R_{q}\right\rangle
$$


Thus the assertion follows once we show that $\left\langle R_{p}, R_{q}\right\rangle=\mathbb{P}^{M}$. By contradiction, suppose that there is a hyperplane $h$ of $\mathbb{P}^{M}$ containing both $R_{p}$ and $R_{q}$. Then, according to Remark 4.5 (ii), $h$ cuts $Y$ along a divisor $D=2 F_{p}+2 F_{q}+R$, with $R$ linearly equivalent to $\Sigma+\beta F$, for some integer $\beta \geq 0$. Then, dotting with $C_{n}$ and recalling (9), we get

$$
\operatorname{deg} C_{n}=D C_{n}=4+\beta \geq 4,
$$

a contradiction.

A further property of $\mathcal{D}_{G}$ is that it is degenerate in $\mathbb{P}^{N \vee}$. In fact, for any $x \in G, \operatorname{Osc}_{x}^{2}(X)$ contains the lines $C_{1}, \ldots, C_{s}$, and hence their linear span $\Lambda:=\left\langle C_{1}, \ldots, C_{s}\right\rangle$ which is a $\mathbb{P}^{2 s+1}$. By duality, this means that $\mathcal{D}_{G}$ is contained in the linear subspace $\mathbb{P}^{N-2 s} \subset \mathbb{P}^{N \vee}$ parameterizing the hyperplanes containing $\Lambda$. Moreover, $\left\langle\mathcal{D}_{G}\right\rangle=\mathbb{P}^{N-2 s}$.

Next we want to determine the degree of $\mathcal{D}_{G}$ when $G$ is of type (2). To do that, recall that $\left\langle C_{i}\right\rangle=\mathbb{P}^{r_{i}}$, and let $d_{i}=\operatorname{deg} C_{i}$

Proposition 4.6 Let $G$ be an irreducible component of $\Phi_{2}(X)$ of type $(2)$. Then $\operatorname{deg} \mathcal{D}_{G}=2 \sum_{i=s+1}^{n}\left(d_{i}-1\right)$.

Proof. Since $G$ is of type (2) we know that $d_{1}=\cdots=d_{s}=1$ and $d_{i} \geq 2$ for $i>s$. Also $r_{1}=\cdots=r_{s}=1$ and $r_{i} \geq 2$ for $i>s$. Recalling that $N=\sum_{i=1}^{n} r_{i}+n-1$, we note that

$$
\operatorname{dim} \mathcal{D}_{G}=N-2 n+1=\sum_{i=1}^{n} r_{i}-n=\sum_{i=s+1}^{n}\left(r_{i}-1\right)
$$

So $\operatorname{deg} \mathcal{D}_{G}$ is the number of elements of $\mathcal{D}_{G}$ contained in a linear system $\mathcal{S} \subset|V|$ defined by $\sum_{i=s+1}^{n}\left(r_{i}-1\right)$ linear conditions, general enough. Choose $r_{i}-1$ general points in each $\mathbb{P}^{r_{i}}$ for $i=s+1, \ldots, n$ and call $Z_{i} \subset \mathbb{P}^{r_{i}}$ the linear subspace they generate. Let $\mathcal{S}$ be the linear system of hyperplanes of $\mathbb{P}^{N}$ defined by the condition of passing through all these points. Let $H \in \mathcal{D}_{G}$ be a hyperplane of $\mathbb{P}^{N}$ not containing $\mathbb{P}^{r_{i}}$ for a given $i, s+1 \leq i \leq n$. Then $h_{i}:=H \cap \mathbb{P}^{r_{i}}$ is a hyperplane of $\mathbb{P}^{r_{i}}$ tangent to the curve $C_{i}$. More precisely, if $H \supset \operatorname{Osc}_{x}^{2}(X)$ and $x \in f_{p}$, then $\operatorname{Osc}_{x}^{2}(X) \supset \operatorname{Osc}_{p_{i}}^{1}\left(C_{i}\right)$, and so $h_{i}$ is tangent to $C_{i}$ at $p_{i}$. On the other hand, if our $H$ is also in $\mathcal{S}$, then, in particular, $h_{i}$ contains $Z_{i}$. Conversely, suppose that $h_{i}$ is a hyperplane of $\mathbb{P}^{r_{i}}$ containing $Z_{i}$ and tangent to $C_{i}$ at a point $p_{i}$, and set

$$
H:=\left\langle C_{1}, \ldots, C_{i-1}, h_{i}, C_{i+1}, \ldots, C_{n}\right\rangle .
$$

Clearly $H$ is a hyperplane of $\mathbb{P}^{N}$. Moreover, $H \in \mathcal{D}_{G}$, because $H$ contains all $\left\langle C_{j}\right\rangle$ for $j \neq i$ and also Osc $p_{p_{i}}^{1}\left(C_{i}\right)$. Furthermore, $H \in \mathcal{S}$ since $H \supset Z_{i}$ for every $i=s+1, \ldots, n$. It thus follows that

$$
\operatorname{deg} \mathcal{D}_{G}=\sum_{i=s+1}^{n} b_{i}
$$

where $b_{i}$ is the number of hyperplanes of $\mathbb{P}^{r_{i}}$ containing $Z_{i}$, that are tangent to $C_{i}$. To compute $b_{i}$ note that $\operatorname{dim} Z_{i}=r_{i}-2$, so $Z_{i}$ is the axis of a pencil of hyperplanes of $\mathbb{P}^{r_{i}}$. The number of hyperplanes in this pencil that are tangent to $C_{i}$ is that of the ramification points of the morphism $C_{i} \rightarrow \mathbb{P}^{1}$ defined by the projection of $C_{i}$ from $Z_{i}$. Thus the Riemann-Hurwitz formula tells us that $b_{i}=2\left(d_{i}-1\right)$ and this concludes the proof.

Relying on the above results we get the following characterization.

Theorem 4.7 Let $X \subset \mathbb{P}^{N}$ be a decomposable scroll generated by $C_{1}, \ldots, C_{n}$, and let $d_{i}=\operatorname{deg} C_{i}$, for $i=1, \ldots, n$. Suppose that $G$ is an irreducible component of type $(2)$ of $\Phi_{2}(X)$. Then $\mathcal{D}_{G}$ is a rational normal scroll if and only if, up to reordering the curves, $d_{1}=\cdots=d_{s}=1$ and $d_{s+1}=\cdots=d_{n}=2$ for some $s \geq 1$.

Proof. As $G$ is of type (2), we can assume that $d_{1}=\cdots=d_{s}=1$ for some $s \geq 1$ and $d_{i} \geq 2$ for $i \geq s+1$, by Proposition 4.2. As we noted, $\mathcal{D}_{G}$ has dimension $N-2 n+1$ and is non-degenerate in $\mathbb{P}^{N-2 s}$. Thus, recalling Proposition 4.6 , the inequality $\operatorname{deg} \mathcal{D}_{G} \geq \operatorname{codim} \mathcal{D}_{G}+1$ becomes

$$
2 \sum_{i=s+1}^{n}\left(d_{i}-1\right) \geq 2(n-s)
$$


Note that this is an equality if and only if

$$
d_{i}=2 \text { for } i=s+1 \ldots, n \text {. }
$$

So, if $\mathcal{D}_{G}$ is a rational normal scroll, then (11) holds. On the other hand, if (11) holds then we know that $X$ is a rational scroll, by Proposition 4.4, and then equality in (10) says that it is normal.

\section{A general lower bound}

In [6, Theorem A] it is shown that the highest inflectional locus of an indecomposable linearly normal elliptic scroll of invariant $e=-1$ is empty. By adapting the argument used in [6] we can locate the highest inflectional locus of an elliptic indecomposable scroll of invariant $e=0$, which is linearly normally embedded. Let $C$ be a smooth curve of genus 1 and let $S=\mathbb{P}(\mathcal{E})$, where $\mathcal{E}$ is the holomorphic rank-2 vector bundle on $C$ defined by the non-split extension

$$
0 \rightarrow \mathcal{O}_{C} \rightarrow \mathcal{E} \rightarrow \mathcal{O}_{C} \rightarrow 0
$$

Let $\pi: S \rightarrow C$ be the ruling projection and denote by $C_{0}$ the tautological section on $S$. Let $\delta \in \operatorname{Div}(C)$ be a divisor of degree $\operatorname{deg} \delta=m+1 \geq 3$ and set $L:=\mathcal{O}_{S}\left(C_{0}+\pi^{*} \delta\right)$. Note that $L$ is very ample, because $\operatorname{deg} \delta \geq e+3$ [4, Ex. 2.12(b), p. 385] and the morphism given by $|L|$ embeds $S$ as a linearly normal scroll of degree $2 m+2$ in $\mathbb{P}^{N}$, where $N=2 m+1$ (note that $m=\frac{N-1}{2}=\left[\frac{N-1}{2}\right]$ ). Let $x \in S$. By [6, $\left.\left(1.0_{m}\right)\right]$ we have

$$
\operatorname{dim}\left(\operatorname{Osc}_{x}^{m}(S)\right)=N-1-\operatorname{dim}(|L-(m+1) x|)=2 m-\operatorname{dim}(|L-(m+1) x|) .
$$

On the other hand, by [6, Remark 1.2] we know that

$$
|L-(m+1) x|=m f_{x}+\left|L-m f_{x}-x\right|,
$$

where $f_{x}$ is the fibre through $x$. Note that the line bundle $L \otimes \mathcal{O}_{S}\left(-m f_{x}\right)=\mathcal{O}_{S}\left(C_{0}+\pi^{*}(\delta-m \pi(x))\right.$ is not necessarily spanned, because $\operatorname{deg}(\delta-m \pi(x))=m+1-m=1<e+2$ [4, Ex. 2.12(a), p. 385]. We have that

$$
\operatorname{dim}\left(\left|L-m f_{x}-x\right|\right)=\operatorname{dim}\left(\left|L-m f_{x}\right|\right)-1 \text { if and only if } L \otimes \mathcal{O}_{S}\left(-m f_{x}\right) \text { is spanned at } x .
$$

Now, twisting (12) by $\mathcal{O}_{C}(\delta-m \pi(x))$ we immediately see that

$$
h^{0}\left(L-m f_{x}\right)=h^{0}\left(\mathcal{E}(\delta-m \pi(x))=2 h^{0}\left(\mathcal{O}_{C}(\delta-m \pi(x))=2 .\right.\right.
$$

Hence (15) gives

$$
\operatorname{dim}\left(\left|L-m f_{x}-x\right|\right)=0 \quad \text { if and only if } L \otimes \mathcal{O}_{S}\left(-m f_{x}\right) \text { is spanned at } x
$$

and taking into account (14) and (13) we get

$$
\operatorname{dim}\left(\operatorname{Osc}_{x}^{m}(S)\right)=2 m \quad \text { if and only if } L \otimes \mathcal{O}_{S}\left(-m f_{x}\right) \text { is spanned at } x .
$$

This proves the following

Proposition 5.1 Let $S \subset \mathbb{P}^{2 m+1}$ be a linearly normal surface scroll over an elliptic curve $C$, defined by an indecomposable vector bundle as in (12), and let $L$ be the hyperplane bundle. Then $x \in \Phi_{m}(S)$ if and only if the line bundle $L \otimes \mathcal{O}_{S}\left(-m f_{x}\right)$ is not spanned at $x$, where $f_{x}$ is the fibre of $S$ through $x$.

Now let $S \subset \mathbb{P}^{N}$ be any surface scroll. Though $S$ can be not decomposable, according to Example 3.2 it seems natural to ask whether, under some assumption, we can get a global lower bound for the dimension of $\operatorname{Osc}_{x}^{k}(S)$, i. e., a lower bound holding at every point $x \in S$, bigger than 3 . We determine such a lower bound, depending on $k$, under certain assumptions on the linear system (not necessarily complete) giving rise to the embedding.

In the following we use the same notation as in [6]. 
Theorem 5.2 Let $S \subset \mathbb{P}^{N}$ be a surface scroll embedded by $|V|$, where $V \subseteq H^{0}(S, L), L=\left.\mathcal{O}_{\mathbb{P}^{N}}(1)\right|_{S}$, and suppose that $N \geq 2 k$. Let $x \in S$ and denote by $f_{x}$ the fibre of $S$ through $x$. If $\left|V-t f_{x}\right|$ is very ample for every non-negative integer $t \leq k-2$, then

$$
\operatorname{dim}\left(\operatorname{Osc}_{x}^{k}(S)\right) \geq k+2 \quad \text { for } k \geq 3 .
$$

Note that this global lower bound is the same holding for rational normal scrolls, as shown by Example 3.1.

Proof. First let us prove, by induction, that

$$
\operatorname{dim}\left(\operatorname{Osc}_{x}^{k}(S)\right) \geq k+1 \quad \text { for any } k \geq 2 .
$$

For $k=2$ this comes from [6. Theorem B] (noting that $N \geq 4$ is enough in the proof). So let $k \geq 3$ and set $\mathcal{L}=L-(k-2) f_{x}$ and $|W|=\left|V-(k-2) f_{x}\right|$. Note that $|W|$ is very ample by assumption and that $S$ embedded by $|W|$ is also a scroll. Actually, for every fibre $f$ of $S$ we have

$$
\mathcal{L} f=\left(L-(k-2) f_{x}\right) f=L f=1 .
$$

Thus [6, Lemma (1.4) and Lemma (1.5)] imply the following facts. The linear system $\left|W-f_{x}\right|$ is base-point free and if $\varphi_{x}: S \rightarrow \mathbb{P}^{M}$ denotes the associated morphism then one of the following conditions holds:

i) every fibre of $\varphi_{x}$ intersects any fibre $f$ of $S$ at a finite set,

ii) $(S, \mathcal{L})=\left(\mathbb{P}^{1} \times \mathbb{P}^{1}, \mathcal{O}_{\mathbb{P}^{1} \times \mathbb{P}^{1}}(1,1)\right)$ and $W=H^{0}(S, \mathcal{L})$.

If ii) holds, then $L=\mathcal{O}(1, k-1)$, hence $h^{0}(L)=2 k$, which implies that

$$
N=\operatorname{dim}(|V|) \leq \operatorname{dim}(|L|)=2 k-1,
$$

but this contradicts our assumption that $N \geq 2 k$. Therefore condition i) holds. Now suppose, by contradiction, that (16) is not true, i. e., $\operatorname{dim}\left(\operatorname{Osc}_{x}^{k}(S)\right) \leq k$. Because $\left|V-(k-3) f_{x}\right|$ is very ample, by induction we know that $\operatorname{dim}\left(\operatorname{Osc}_{x}^{k-1}(S)\right) \geq k$. So, due to the obvious inclusion $\operatorname{Osc}_{x}^{k}(S) \supseteq \operatorname{Osc}_{x}^{k-1}(S)$ we conclude that

$$
\operatorname{Osc}_{x}^{k}(S)=\operatorname{Osc}_{x}^{k-1}(S) \text {. }
$$

Equivalently, this says that

$$
|V-(k+1) x|=|V-k x| .
$$

This in turn, according to [6, Remark (1.2)], implies the equality

$$
\operatorname{dim}\left(\left|V-k f_{x}-x\right|\right)=\operatorname{dim}\left(\left|V-(k-1) f_{x}-x\right|\right) .
$$

Hence

$$
f_{x} \subseteq \mathrm{Bs}\left(\left|V-(k-1) f_{x}-x\right|\right)=\mathrm{Bs}\left(\left|W-f_{x}-x\right|\right)=\varphi_{x}^{-1}\left(\varphi_{x}(x)\right) .
$$

But this contradicts condition i). To conclude the proof we show that equality cannot occur in (16) for $k \geq 3$. First of all, since $\left|V-t f_{x}\right|$ is very ample for all $t \leq k-2$, by applying [6, Remark (1.7)] inductively we see that

$$
\operatorname{dim}(|W|)=\operatorname{dim}(|V|)-2(k-2)=N-2(k-2) .
$$

Note that

$$
\operatorname{dim}(|V-(k+1) x|)=\operatorname{dim}(|W-3 x|)
$$

by [6. Remark (1.2)]. Due to (16) and the assumption $N \geq 2 k, S$ is embedded by $|W|$ as a scroll in a projective space of dimension $\geq 4$; hence $|W-3 x| \neq|W-2 x|$ by [6, Theorem B]. Since $|W|$ is very ample this says that $\operatorname{dim}(|W-3 x|)<\operatorname{dim}(|W|)-3$. Thus, recalling the equality

$$
\operatorname{dim}(|V-(k+1) x|)+\operatorname{dim}\left(\operatorname{Osc}_{x}^{k}(S)\right)=N-1,
$$

we get

$$
\operatorname{dim}\left(\operatorname{Osc}_{x}^{k}(S)\right)>N+2-\operatorname{dim}(|W|) .
$$

Finally, combining (17) with (18) and assuming equality in (16) gives $k \leq 2$. This completes the proof. 
Acknowledgements During the preparation of this paper the first author has been supported by the MUR of the Italian Government in the framework of the PRIN "Geometry on Algebraic Varieties", and the second author by the projects BFM200303917/MATE (Spanish Ministry of Education) and Santander/UCM PR27/05-13876. The first author would also like to thank the GNSAGA-INDAM and Azione Integrata Italia-Spagna IT200 for support received at an early stage of this research. Both authors are grateful to the University of Milan for financial support.

\section{References}

[1] E. Arbarello, M. Cornalba, Ph. Griffiths, and J. Harris, Geometry of Algebraic Curves, Vol. I (Springer, New York, 1985).

[2] E. Ballico and C. Fontanari, On the osculatory behaviour of higher dimensional projective varieties, Collect. Math. 55, 229-236 (2004).

[3] E. Ballico, R. Piene, and H. Tai, A characterization of balanced rational normal surface scrolls in terms of their osculating spaces, II, Math. Scand. 70, 204-206 (1992).

[4] R. Hartshorne, Algebraic Geometry (Springer, New York, 1977).

[5] T. Johnsen, Plane projections of a smooth space curve, Parameter Spaces, Banach Center Publications, vol 36 (1996) pp. 89-110.

[6] A. Lanteri, On the osculatory behavior of surface scrolls, Le Matematiche (Catania) 55, 447-458 (2000).

[7] R. Mallavibarrena and R. Piene, Duality for elliptic normal surface scrolls, Enumerative Algebraic Geometry, edited by S. L. Kleiman and A. Thorup, Contemporary Mathematics, vol. 123, (Amer. Math. Soc., 1991), pp. 149-160.

[8] R. Piene, Numerical characters of a curve in projective $n$-space, Real and Complex Singularities, edited by P. Holm (Sijthoff and Noordhoff, Groningen, 1978), pp. 475-496.

[9] R. Piene and G. Sacchiero, Duality for rational normal scrolls, Comm. Algebra 12, 1041-1066 (1984).

[10] R. Piene and H. S. Tai, A characterization of balanced rational normal scrolls in terms of their osculating spaces, Enumerative Geometry, Proc. Sitges, 1987 edited by S. Xambo-Descamps, Lecture Notes in Math., vol 1436, (Springer, 1990), pp. 215-224.

[11] T. Shifrin, The osculatory behavior of surfaces in $\mathbb{P}^{5}$, Pacif. J. Math. 123, 227-256 (1986). 